\title{
Calcul de probabilités de dépassement d'objectifs environnementaux de rejets de sources ponctuelle et diffuse à l'aide du système de modélisation intégrée GIBSI Computation of probability of exceeding environmental load allocations from point and diffuse sources using the integrated modeling system GIBSI
}

\author{
A. N. Rousseau, A. Mailhot, S. Gariépy, E. Salvano et J. P. Villeneuve
}

Volume 15, numéro hors-série, 2002

URI : https://id.erudit.org/iderudit/705490ar

DOI : https://doi.org/10.7202/705490ar

Aller au sommaire du numéro

\section{Éditeur(s)}

Université du Québec - INRS-Eau, Terre et Environnement (INRS-ETE)

ISSN

0992-7158 (imprimé)

1718-8598 (numérique)

Découvrir la revue

Citer cet article

Rousseau, A. N., Mailhot, A., Gariépy, S., Salvano, E. \& Villeneuve, J. P. (2002). Calcul de probabilités de dépassement d'objectifs environnementaux de rejets de sources ponctuelle et diffuse à l'aide du système de modélisation intégrée GIBSI. Revue des sciences de l'eau / Journal of Water Science, 15, 121-148. https://doi.org/10.7202/705490ar

\section{Résumé de l'article}

Dans un contexte de gestion intégrée de l'eau par bassin versant, on doit, avant d'implanter tout programme d'assainissement, comparer les solutions de rechange, distinguer les contributions des différentes sources de pollution aux problèmes de qualité de l'eau, et définir des objectifs environnementaux de rejet (OER) associés aux activités et aux pressions anthropiques. Dans le cas des sources de pollution d'origine ponctuelle, on détermine un OER et son risque de dépassement en fonction d'un débit d'étiage critique d'une période de retour donnée. Dans le cas de la pollution diffuse d'origine agricole, il n'existe pas de concepts similaires pour définir des OER. L'approche que nous proposons, c'est de simuler, à l'aide de chroniques météorologiques et de divers scénarios de gestion de ces rejets, les concentrations résultantes dans les cours d'eau et de calculer les probabilités de dépassement des critères de qualité de l'eau (CQE). Cette approche permet de caractériser la prise de décision pour la période de contrôle en matière du nombre moyen de jours de dépassement d'un CQE. Ceci représente une quantification plus exacte du risque car ce dernier est interprété en fonction de ce qui est mesuré en rivière, c'est-à-dire les concentrations de polluants. Dans cette étude, on illustre l'application de ce concept à partir d'un cas de figure dans le bassin versant de la rivière Chaudière (Québec, Canada) qui est simulé avec le système de modélisation intégrée GIBSI. Dans le cadre d'une analyse de scénarios de restauration des activités récréatives conduisant à des contacts directs et fréquents avec l'eau, on illustre comment on peut, en réduisant respectivement les pressions agricole et urbaine de 32 et $17 \%$, abaisser globalement les probabilités de dépassement des critères esthétique et bactériologique de 0,32 à 0,19 et de 0,94 à 0 , respectivement. 


\title{
Calcul de probabilités de dépassement d'objectifs environnementaux de rejets de sources ponctuelle et diffuse à l'aide du système de modélisation intégrée GIBSI
}

\author{
Computation of probability of exceeding \\ environmental load allocations from point and diffuse \\ sources using the integrated modeling system GIBSI
}

\author{
A.N. ROUSSEAU ${ }^{1}$, A. MAILHOT ${ }^{1}$, S. GARIÉPY ${ }^{2}$, E. SALVANO ${ }^{1}$, \\ J.-P. VILLENEUVE ${ }^{1}$
}

SUMMARY

In an integrated watershed management context, the implementation of any clean water program requires the evaluation of the contribution of pollutant loads associated with wet (nonpoint or diffuse) and dry weather (point) sources to the studied water quality problem. It is also necessary to set environmental load allocations $(E L A)$ or total maximum daily loads $(T M D L)$ for various anthropogenic activities and to link these loads to water pollutant concentrations. The $E L A$ or $T M D L$ is a numerical quantity determining the maximum load of pollutants from point and nonpoint sources as well as background sources, to receiving water bodies that will meet designated water uses (e.g., swimming or fishing) in terms of water quality standards (WQS). The American Congress included a $T M D L$ program in the Clean Water Act of 1972 (US EPA, 1997; 1999). The outcome of the TMDL program corresponds to the drafting of a watershed management plan (NOVOTNY, 1999).

Estimation of ELA from point sources is generally based on simultaneous occurrence of severe low flows and maximum daily loads. The ensuing risk of not meeting WQS is usually linked to the inverse of the return period of the design flow. Meanwhile, estimation of $E L A$ from diffuse sources, and associated environmental risks, is not as well established since diffuse sources of pollution generally occur during important runoff events and strongly depend on land use and management practices. These wet weather loads may be allocated using continuous hydrological modelling. Simulation results can then in return be used to link $E L A$ from point sources and diffuse sources to pollu-

1. Université du Québec, INRS, Centre Eau, Terre et Environnement, 2800 rue Einstein, Sainte-Foy, QC (Canada) G1V 4C7.

2. Ministère de l'Environnement du Québec, Direction des politiques du secteur agricole, 675, RenéLévesque Est, 8 étage, boîte 26, Québec, QC (Canada) G1R $5 V 7$.

* Correspondance. E-mail : alain_rousseau@inrs-eau.uquebec.ca

** Les commentaires seront reçus jusqu'au 30 avril 2003. 
tant concentrations and, for a given time period (e.g., summer), to evaluate the probabilities (namely the risks) of exceeding WQS designating the water use.

The objective of this study was to present, using the integrated modelling system GIBSI (VILLENEUVE et al., 1998b; MAILHOT et al., 1997), this new approach to assess the risk associated with the establisment of $E L A$ from point and diffuse sources. A case study on the Chaudière River watershed (Quebec, Canada) was defined and simulations were performed (scenarios AE). The case study focussed on determining whether WQS defining the designated recreational use of water requiring direct and prolonged contact were attainable. Untreated municipal waste waters from a small town (St. Martin) and nonpoint source pollution were responsible for impairment of the studied river segment. Water contaminants considered were fecal coliforms $(F C)$ and phosphorus $(P)$.

Two base case scenarios, $A$ and $C$, were simulated using four years (19821985) of meteorological data to illustrate the degraded bacteriological and aesthetic conditions of the river segment due to dry and wet weather sources, respectively. Dry weather sources were assumed to solely contribute to the bacteriological impairment. Meanwhile, both wet and dry weather sources were assumed to contribute to aesthetic impairment. Scenario B was defined to examine the impact of constructing a waste water treatment plant (WWTP) for the town of St. Martin on the bacteriological conditions. Scenarios $D$ and $E$ were elaborated to quantify the impact of both reducing diffuse and point source loads on aesthetic conditions, respectively. For scenario $\mathrm{C}$, on average $31 \mathrm{~kg} P / \mathrm{ha}$ were applied on cultivated land according to local fertilization calendars. Similarly, the fertilization rate for scenarios $D$ and $E$ was on average $13 \mathrm{~kg} P / \mathrm{ha}$. The design stream flow for $E L A$ from point sources was $a_{30} Q_{5}$ (30 day low flow, 5 year return period) and estimated at $6.05 \mathrm{~m}^{3} / \mathrm{s}$ for the studied river segment. It is noteworthy to mention that for the 19821985 summers (June 21 through September 20), simulated daily stream flows exceeded the design flow $31,62,19$ and 2 times and that 150, 48, 348, and $270 \mathrm{Mm}^{3}$ of water flowed through the studied river segment, respectively. This means the $\mathbf{1 9 8 3}$ meteorological series was three times as dry as that of 1982 despite the fact it was the latter that produced the ${ }_{30} Q_{5}$. The $E L A$ from both point and diffuse sources for the river segment receiving the St. Martin's effluent and the studied river segment were $0.75 \cdot 10^{12} U F C$ and $11.21 \mathrm{~kg}$ $P$ and $1.05 \cdot 10^{12} U F C$ and $15.68 \mathrm{~kg} P$, respectively.

For scenario A, simulation results showed that respectively $94 \%$ and $14 \%$ of the time, the bacteriological $\left(W Q S_{F C}<200 \mathrm{UFC} / 100 \mathrm{~mL}\right.$ ) and aesthetic $\left(W Q S_{P}<0,03 P \mathrm{mg} / L\right) W Q S$ were not met over the summer season of the four-year meteorological series. Similarly, for scenario $\mathrm{C}$, the simulation results indicated that $32 \%$ of the time, the aesthetic WQS was not achieved. Dry weather sources, namely WWTP and wet weather sources, namely agricultural runoff, accounted for $P$ loads. During the driest summer, dry weather sources accounted for $63 \%$ of the $P$ loads. For the other summers, wet weather sources accounted for 71,88 , and $78 \%$ of total $P$ loads. For scenario B, simulation results showed that the bacteriological WQS was met $100 \%$ of the time. Meanwhile, scenario D was designed to see whether a $27 \%$ reduction of the agricultural nonpoint source load could alone restore the aesthetic properties. The simulation results showed an improvement as the probability of exceedence dropped from 0.32 to 0.27 . This probability was further lowered to 0.19 when St. Martin's waste waters were treated using aerated lagoons and dephosphotation. This corresponded to an additional $17 \%$ abatement of the dry weather sources loads (scenario E).

It is noteworthy that this study did not specifically attempt to specify the reasons why WQS were not met, whether it was due to low stream flows or large point or nonpoint sources loads although the integrated modelling system 
allows for this. Also, this study did not attempt to determine impacts of local management scenarios instead of a systematic reduction though GIBSI also allows for this. However, this study clearly illustrated how the proposed methodology, which is rooted in a risk assessment approach based on evaluation of the probability of exceeding WQS, is well suited to characterise $E L A$ for various anthropogenic activities. Indeed, simulation results clearly demonstrated the benefits of assessing independently the impacts of $E L A$ from point and diffuse sources on the attainability of a designated water use. It is believed that this type of $E L A$ assessment approach will facilitate communication with stakeholders. Ideally, these exceeding probabilities should be evaluated using long meteorological series (e.g., 30 years).

Key-words: integrated watershed management, point and nonpoint/diffuse sources of pollution, probability of exceeding water quality standards, integrated modelling system.

\section{RÉSUMÉ}

Dans un contexte de gestion intégrée de l'eau par bassin versant, on doit, avant d'implanter tout programme d'assainissement, comparer les solutions de rechange, distinguer les contributions des différentes sources de pollution aux problèmes de qualité de l'eau, et définir des objectifs environnementaux de rejet $(O E R)$ associés aux activités et aux pressions anthropiques. Dans le cas des sources de pollution d'origine ponctuelle, on détermine un $O E R$ et son risque de dépassement en fonction d'un débit d'étiage critique d'une période de retour donnée. Dans le cas de la pollution diffuse d'origine agricole, il n'existe pas de concepts similaires pour définir des $O E R$. L'approche que nous proposons, c'est de simuler, à l'aide de chroniques météorologiques et de divers scénarios de gestion de ces rejets, les concentrations résultantes dans les cours d'eau et de calculer les probabilités de dépassement des critères de qualité de l'eau $(C Q E)$. Cette approche permet de caractériser la prise de décision pour la période de contrôle en matière du nombre moyen de jours de dépassement d'un $C Q E$. Ceci représente une quantification plus exacte du risque car ce dernier est interprété en fonction de ce qui est mesuré en rivière, c'est-à-dire les concentrations de polluants. Dans cette étude, on illustre l'application de ce concept à partir d'un cas de figure dans le bassin versant de la rivière Chaudière (Québec, Canada), qui est simulé avec le système de modélisation intégrée GIBSI. Dans le cadre d'une analyse de scénarios de restauration des activités récréatives conduisant à des contacts directs et fréquents avec l'eau, on illustre comment on peut, en réduisant respectivement les pressions agricole et urbaine de 32 et $17 \%$, abaisser globalement les probabilités de dépassement des critères esthétique et bactériologique de 0,32 à 0,19 et de 0,94 à 0 , respectivement.

Mots-clés : gestion intégrée des bassins versants, pollutions ponctuelle et diffuse d'origines agricole et municipale, objectifs environnementaux de rejet, qualité de l'eau, probabilités de dépassement de critères de qualité de l'eau.

\section{1 - INTRODUCTION}

Au cours des vingt dernières années, le gouvernement du Québec (1997) a investi en capital et intérêts près de 15,3 milliards de dollars [2 100 \$ per capita, en supposant 7,3 millions d'habitants (Statistiques Canada, 1999)] pour 
contrôler les sources ponctuelles ciblées ${ }^{1}$ par les Programmes d'assainissement des eaux du Québec (PAEQ, 1978-1994) et des eaux municipales (PADEM, 1995-1999). L'analyse de PAINCHAUD (1997a, b) des chroniques du Réseau rivières révèle que ces programmes ont eu des effets positifs sur la qualité de l'eau au niveau de la $D B O_{5}$, des coliformes fécaux (CF) et des MES en aval des stations de traitement des eaux usées au cours de la période 19791994. Dans les bassins versants agricoles, il y a eu une tendance à la hausse des concentrations de $\mathrm{N}-\mathrm{NO}_{3}^{-}$- bien que celles-ci soient demeurées inférieures à $10 \mathrm{mg} \mathrm{N}-\mathrm{NO}_{3}^{-} / \mathrm{L}$, critère pour l'eau brute destinée à la consommation humaine (Ministère de l'Environnement du Québec, 1990, rév. 1992) - et une tendance à la baisse des concentrations de $P$ - quoique celles-ci affichaient de fortes médianes estivales, entre 0,1 et $0,2 \mathrm{mg} P / L$, largement supérieures au critère de $0,03 \mathrm{mg} P / L$ pour la prévention de l'eutrophisation des rivières (Ministère de l'Environnement du Québec, 1990, rév. 1992). GANGBAZO (1996) et NOLET et al. (1999) estiment qu'en moyenne $73,48,34$ et $76 \%$ des charges de $N$ ainsi que $75,52,56$ et $63 \%$ des charges de $P$ des flux nets à l'exutoire des rivières Yamaska, l'Assomption, Chaudière et Boyer proviendraient des activités agricoles. Ce constat suggère que si l'on veut atteindre l'objectif du $P A E Q$ et du PADEM, il faut maintenant s'attaquer à la pollution diffuse en milieu agricole (GANGBAZO, 1997 ; PAINCHAUD 1997a, b ; GANGBAZO et PAINCHAUD, 1999).

Dans un contexte de gestion intégrée de l'eau par bassin versant, on doit, avant d'implanter d'autres programmes d'assainissement, comparer les solutions de rechange, distinguer les contributions des différentes sources de polIution aux problèmes de qualité de l'eau, et définir des objectifs environnementaux de rejet $^{2}(O E R$ ) associés aux activités anthropiques. Pour le secteur agricole, NOLET et al. (1999) ont défini deux catégories d'objectifs environnementaux :

- ceux visant la réduction ou l'atténuation globale des pressions (ex. : l'objectif réglementaire sur la réduction de la pollution d'origine agricole (RRPOA) de juillet 1997 sur l'installation d'infrastructures d'entreposage des déjections animales, la réalisation d'un plan agroenvironnemental de fertilisation basé sur les besoins agronomiques et la mise en place de techniques de travail de sol minimisant la pollution diffuse) ;

- ceux visant à réduire ou atténuer les pressions par bassin ou sous-bassin versant en tenant compte des particularités locales et régionales. Cette dernière catégorie cherche à établir un lien entre les rejets et les concentrations dans le cours d'eau.

MICHAUD et al. (2001) ont récemment présenté le développement et la validation d'indicateurs de risque de perte de $P$ qui s'inscrivent dans cette deuxième catégorie d'objectifs. La première catégorie d'objectifs, basée sur le concept du développement durable, s'inspire d'une approche préventive sans évaluation directe quant aux impacts à l'échelle du bassin versant. La deuxième catégorie

1. Assainissement des eaux usées municipales, traitement des effluents des fabriques de pâtes et papiers et construction de structures d'entreposage des fumiers et lisiers.

2. De manière générale, ces objectifs cherchent à maintenir les activités humaines à des niveaux sans effets graves ou irréversibles sur les écosystèmes, à réduire ou limiter les pressions à un niveau acceptable pour les écosystèmes et les usages associés, ou encore à protéger, récupérer ou restaurer les écosystèmes (NOLET et al., 1999). 
s'articule plutôt autour de la démarche de gestion intégrée qui tient compte de l'ensemble des sources de polluants susceptibles d'affecter la qualité du milieu aquatique (ROUSSEAU et al., 2000a).

Dans le cas des sources de pollution d'origine ponctuelle, on détermine un $O E R$ en considérant un débit d'étiage critique d'une période de retour donnée. Dans le cas de la pollution diffuse d'origine agricole, il n'existe pas d'approches équivalentes permettant de définir des $O E R$. La pratique générale est d'évaluer sur une base annuelle la charge moyenne d'un polluant à la sortie d'un bassin versant ou d'un sous-basin versant à partir de données observées de qualité de l'eau et de déterminer le pourcentage de réduction nécessaire au respect moyen du critère de qualité de l'eau pour un paramètre donné (Ministère de l'Environnement et de la Faune du Québec, 1995). L'utilisation de conditions moyennes pour établir ce taux de réduction reste cependant une première approximation. Si l'on cherche à développer un cadre de définitions des OER d'origine diffuse s'inspirant de celui utilisé pour les OER d'origine ponctuelle, il faut considérer un événement météorologique causant un ruissellement de surface et/ou un écoulement hypodermique important (débit de crue critique d'une période de retour donnée) puisque ces conditions sont essentielles à l'apport d'une pollution diffuse. Quant à la définition et au suivi de normes de rejets diffus, il demeure très difficile de faire la caractérisation des charges produites à l'échelle des champs. Ceci requerrait beaucoup trop de campagnes de suivi. Ce que nous proposons, c'est de simuler, à l'aide de chroniques météorologiques et de divers scénarios d'intervention, les concentrations résultantes dans les cours d'eau et de calculer les probabilités de dépassement des critères de qualité. Cette approche permettrait de caractériser la prise de décision en matière du nombre moyen de jours de dépassement, ce qui peut être plus utile au gestionnaire que d'interpréter un scénario sur la base d'une période de retour annuelle. À noter ici que l'on ne remet pas en question la détermination d'un $O E R$ d'origine ponctuelle, défini à partir de conditions hydrométriques " sèches " (en période de faible précipitation, choix d'un débit d'étiage critique et d'une concentration critique du rejet). Ce que I'on propose c'est d'élaborer un concept d'OER d'origine diffuse basé sur la simulation de divers scénarii à l'aide de chroniques météorologiques incluant des conditions météorologiques " mouillées " (périodes de précipitations importantes).

L'objectif de cet article est d'illustrer, à partir d'un cas de figure pour le bassin versant de la rivière Chaudière (Québec, Canada), ce cadre d'application du concept d'OER à l'aide du système de modélisation intégrée GIBSI développé par VILLENEUVE et al. (1998b). Plus particulièrement, on utilisera ce concept pour évaluer divers scénarii d'intervention visant la réduction des pressions agricole et urbaine liées à l'augmentation des concentrations en $P$ dans les cours d'eau. De plus, ce concept sera appliqué pour évaluer la probabilité de dépassement d'un OER d'origine ponctuelle associé à la réduction d'une contamination bactériologique issue d'une pression du milieu urbain. 


\section{2 - THÉORIE}

\subsection{OER d'origine ponctuelle}

Le calcul d'un OER porte sur la détermination de la charge tolérable d'un polluant qui peut être rejetée au cours d'eau tout en permettant de protéger ou de restaurer un usage de l'eau (ex. : activités récréatives) à la limite d'une zone de mélange (ex. : $300 \mathrm{~m}$ ). Ce calcul correspond à la différence entre la charge maximale associée au critère de qualité de l'eau (CQE) concerné par l'usage, et la charge en amont du rejet ou de l'effluent (Ministère de l'Environnement, 1991, rév. 2001) :

$$
O E R=Q_{d} \cdot C Q E-Q_{c} \cdot C_{a m}
$$

que l'on peut également exprimer en fonction des caractéristiques du rejet :

$$
O E R=Q_{e} \cdot C_{e}
$$

où

$$
Q_{d}=Q_{e}+Q_{c}
$$

où $Q_{d}$ est le débit de dilution ou de mélange, $Q_{c}$ le débit critique ${ }^{3}$ associé à l'écoulement du cours d'eau, $C_{a m}$ la concentration amont caractérisant le cours d'eau, $Q_{e}$ le débit de l'effluent et $C_{e}$ la concentration de l'effluent. Si l'on isole $C_{e}$ dans l'équation (1a), on peut ainsi spécifier la concentration tolérable à l'effluent. De plus, afin que les grands cours d'eau ne soient pas utilisés à des fins de dilution, on requiert que le rapport $Q_{e} / Q_{d}$, qui correspond au facteur de dilution $\left(F_{d}\right)$, soit plus grand ou égal à 0,01 . Cette approche, qualifiée de locale, permet une protection du milieu aquatique immédiatement en aval d'un rejet.

Pour la résolution d'un problème en aval résultant d'un cumul de charges multiples, il faut considérer l'ensemble des rejets $i$ en amont dans le calcul d'un $O E R$. On a alors recours à une approche globale pour déterminer les $C_{e i}$ (Ministère de l'Environnement, 1991, rév. 2001) :

$$
\sum_{i=1}^{n} Q_{e j} P_{i} C_{e i}=\left(Q_{c}+\sum_{i=1}^{n} Q_{e i}\right) C_{c}-Q_{c} C_{a m}
$$

où $P_{i}$ représente la fraction restante du polluant considérée au tronçon à l'étude. Incidemment, cette fraction est souvent évaluée à l'aide d'une fonction exponentielle ayant pour paramètres une constante de décroissance du polluant et un temps moyen de transport entre l'effluent et l'usage (Ministère de l'Environnement, 1991, rév. 2001). En reconnaissant que l'expression entre parenthèses du côté droit de l'équation (2a) représente $Q_{d}$ et, que dans une première approximation, $Q_{c}$ est du même ordre de grandeur que $Q_{d}$, l'équation devient :

$$
\sum_{i=1}^{n} Q_{e i} P_{i} C_{e i}=Q_{d}\left(C_{c}-C_{a m}\right)
$$

3. Pour les usages requérant des temps d'exposition longs, un débit critique correspondant à un $Q_{2-7}$ (durée de 7 jours période de retour de 2 ans) ou un $Q_{5-30}$, pour la toxicité chronique un débit critique correspondant à un $Q_{10-7}$ (Ministère de l'Environnement, 1991, rév. 2001). 
Ce qui correspond à la charge tolérable. Cependant, on se retrouve avec une équation et $i$ inconnus. Ce problème peut se simplifier par une répartition globale de l'effort de dépollution en exigeant une concentration égale à chaque effluent et un débit critique uniforme sur tout le tronçon de cours d'eau en amont, ainsi, les $C_{e i}$ deviennent égaux et l'équation (2b) est réduite à l'expression suivante :

$$
C_{e}=\frac{Q_{d}\left(C_{c}-C_{a m}\right)}{\sum_{i=1}^{n} Q_{e i} P_{i}}
$$

À noter qu'il serait possible d'optimiser cet effort de dépollution (HAITH, 1982).

\subsection{OER d'origine diffuse}

La détermination d'un OER d'origine diffuse a pour but de résoudre un problème en aval résultant d'un cumul de charges diffuses multiples. Ceci suggère que l'on peut examiner la problématique à partir de l'équation (2c) si on suppose une concentration du même ordre de grandeur pour tous les rejets. La charge tolérable pour chacune des sources diffuses (i.e., champs agricoles) s'obtiendra en utilisant le ruissellement produit à chacune de ces sources. Cette approche soulève cependant quelques problèmes. Tout d'abord, il faut :

- identifier les technologies d'assainissement agricole [i.e., pratiques agricoles de conservation (PAC)] qui permettront d'obtenir la concentration obtenue à l'aide de l'équation (2c) ;

- définir un débit critique qui est caractérisé par un volume de ruissellement appréciable.

De plus, il faut définir des conditions initiales telles que les contenus en eau et en éléments nutritifs du sol qui sont représentatives des cultures et des taux de fertilisation. Ces tâches peuvent devenir très ardues compte tenu de la très grande variabilité des caractéristiques physiques, chimiques et biologiques des champs. Quant à la définition de normes de rejets, il demeure difficile de faire le suivi des charges produites à l'échelle des champs agricoles.

Dans le contexte de la méthodologie proposée, la caractérisation d'un OER d'origine diffuse, requiert, dans un premier temps, de recourir à la modélisation des processus biophysicochimiques responsables de la pollution d'origine diffuse (VILLENEUVE et al., 1998a ; GANGBAZO et al., 1999) et, dans un deuxième temps, de simuler divers scénarios d'intervention en utilisant plusieurs chroniques météorologiques annuelles (NOVOTNY, 1999). L'analyse statistique des résultats de simulations permettra, à l'aide d'une probabilité moyenne de dépassement de l'OER à l'étude, de caractériser la variabilité liée aux aléas météorologiques. Pour une période de l'année pré-sélectionnée (ex. : saison estivale), cette probabilité pourra, par exemple, s'exprimer par le nombre moyen de jours où le critère d'usage de l'eau visé n'est pas satisfait. De plus, cette approche permettra de définir, à l'échelle du bassin versant, des normes de rejets d'origine agricole basées sur l'application, voire simulation des technologies d'assainissement de l'eau usuelles en milieu agricole (ex. : les PAC) et surtout de quantifier les risques associés à leur impact sur l'environnement. Ceci 
est important car, en général, les études de terrain servent à déterminer l'efficacité des PAC à l'échelle de la parcelle expérimentale.

\subsection{OER d'origines ponctuelle et diffuse}

Le concept d'OER d'origines ponctuelle et diffuse s'inspire en grande partie du protocole américain pour les TMDL (Total maximum daily load) de la US EPA (1997, 1999). Le TMDL représente, exprimé en charges de polluants, la charge maximale journalière d'un polluant, incluant la contribution de la pollution diffuse, qu'une section de cours d'eau problématique peut recevoir tout en respectant à long terme l'usage de l'eau à restaurer ou à conserver. L'approche $T M D L$ sous-entend que toutes les sources de pollution ponctuelle et diffuse, de même que les caractéristiques des cours d'eau, sont fondamentalement liées (JARRELL, 1999). Cette façon de faire, qui a été incluse dans la Clean water act de 1972 (voir GARIEPY et ROUSSEAU, 2000), est présentement promue par la US EPA, conjointement avec l'allocation de bornes supérieures de rejets ponctuels du programme NPDES (National pollutant discharge elimination system), pour mettre en place la gestion de l'eau par bassin versant (NOVOTNY et OLEM, 1994 ; SHOEMAKER et al., 1997 ; US EPA, 1997, 1999 ; NOVOTNY, 1999 ; CURREY et KLEINMAN, 1999). Ainsi, le programme TMDL établit des liens entre le contrôle de la pollution diffuse et les rejets ponctuels par le biais de permis d'émission du NPDES. Ce programme encourage aussi l'adoption de meilleures pratiques de gestion (BMP, Best management practice). À noter que tous ces enjeux sont aussi considérés dans le cadre de la politique communautaire de l'eau du parlement européen (Official Journal of the European Communities, 2000).

L'adéquation entre le concept général des OER proposé ici et le programme TMDL de la US EPA peut s'exprimer comme suit :

$$
T M D L=\sum_{j=1}^{n} O E R_{\text {Ponctuelle }_{j}}+\sum_{i=1}^{m} O E R_{{\text {Diffuse } e_{i}}}+Q_{c} \cdot C_{a m}+M S
$$

que l'on peut aussi écrire selon la définition du US EPA de la manière suivante :

$$
T M D L=L C=\sum_{j=1}^{n} W L A_{j}+\sum_{i=1}^{m} L A_{i}+M S
$$

où $L C$ représente la charge maximale qu'un cours d'eau peut recevoir sans enfreindre le critère de qualité de l'eau à l'étude, WLA l'allocation de rejet ou portion du $T M D L$ allouée à une source ponctuelle $j$ existante ou future, $L A$ l'allocation de charge ou portion du $T M D L$ allouée à une source diffuse $i$ existante ou future ainsi qu'à la contribution naturelle du milieu, et $M S$ une marge de sécurité définie comme étant une quantification de l'incertitude sur les relations entre la charge de polluant et la qualité de l'eau du milieu. À noter que l'approche TMDL se veut une approche globale.

Dans le cas d'un tronçon problématique, tronçon où l'on cherche à conserver ou restaurer un usage pour une période donnée, on peut également calculer le pourcentage de réduction, $P R$, de la charge actuelle d'un polluant, $C H A$, qui permettra de satisfaire l'OER dans ce tronçon (GOUIN, 1984 ; Ministère de l'Environnement et de la Faune du Québec, 1995) : 


$$
P R=100\left[\frac{C H A-\left(O E R_{\text {Ponctuelle }}+O E R_{\text {Diffuse }}\right)}{C H A-C H N}\right]
$$

avec

$$
C H N<\left(O E R_{\text {Ponctuelle }}+O E R_{\text {Diffuse }}\right)<C H A
$$

Ce qui est difficile à déterminer c'est la portion diffuse de CHA. En effet, cette dernière est variable dans le temps et en fonction des conditions météorologiques, ce qui n'est pas le cas des rejets ponctuels dont la variabilité temporelle est nettement moins significative. II est aussi important de se rappeler que, contrairement aux rejets d'origine ponctuelle qui se déversent localement, les rejets d'origine diffuse proviennent de l'ensemble du bassin versant délimité par le tronçon à l'étude.

\subsection{Calcul de probabilité de dépassement}

Pour une période donnée de l'année (ex. : saison estivale), on détermine la probabilité de dépassement du critère de qualité de l'eau, $C Q E$, pour l'usage à l'étude de la façon suivante :

$$
P([X]>C Q E)=1-F_{[X]}(C Q E)
$$

où $[X]$ représente la concentration du polluant visé par l'usage de l'eau à l'étude (résultant des charges diffuse et ponctuelle du polluant découlant d'un scénario d'intervention donné) et $F_{[X]}(C Q E)$ est la valeur de la cumulative de la fonction de densité de probabilité de $[X]$ évaluée à la valeur du CQE. À partir de cette caractérisation, on pourra calculer le nombre de jours où le $C Q E$ visé n'est pas satisfait.

\section{3 - SYSTĖME DE MODÉLISATION INTÉGRÉE GIBSI}

Le système de modélisation intégrée GIBSI (Gestion intégrée des bassins versants à l'aide d'un système informatisé) a tous les éléments de base requis pour calculer la probabilité de dépassement d'un OER, toutes contributions ponctuelles et diffuses confondues. Pour une description détaillée ou sommaire du développement du système, voir respectivement VILLENEUVE et al. (1998b) ou MAILHOT et al. (1997). Les modèles mathématiques de simulation de l'hydrologie, de l'érosion des sols, du transport et du devenir de l'azote, du phosphore et des pesticides, ainsi que de la qualité de l'eau en rivière et en lac de GIBSI, permettent d'évaluer les impacts de différents scénarios d'intervention sur la quantité et la qualité de l'eau tout le long du réseau hydrographique.

Le modèle hydrologique de GIBSI est HYDROTEL (FORTIN et al., 1995, $2001 a, b)$. Le modèle d'érosion hydrique des sols repose sur l'utilisation des facteurs des modèles USLE (WISCHMEIER et SMITH, 1978) et RUSLE (RENARD et al., 1997), en y ajoutant certaines particularités issues d'autres modèles (ex. : cheminement des sédiments). La modélisation du transport et du devenir de 
l'azote, du phosphore et des pesticides en milieu terrestre est basée sur les algorithmes du modèle de bassin versant SWRRB (ARNOLD et WILLIAMS, 1995) et du modèle de productivité des sols EPIC de SHARPLEY et WILLIAMS (1990). Le modèle de qualité est QUAL2E (BROWN et BARNWELL, 1987). II est important ici de préciser que les modèles d'érosion, de transport et de qualité de l'eau ont tous été adaptés aux conditions québécoises et aux besoins de la modélisation. Par exemple, le modèle QUAL2E fonctionne en régime transitoire.

L'unité spatiale de simulation pour la qualité de l'eau est le tronçon de cours d'eau d'un sous-bassin versant élémentaire [unité spatiale de simulation (USS) pour le bilan hydrologique] et l'unité temporelle de simulation est la journée. Pour ce qui est des processus d'érosion, de transport et du devenir des polluants agricoles, ils sont simulés pour chacune des zones d'occupation agricole d'une unité spatiale de simulation. Les USS sont définies à l'aide d'un modèle numérique d'altitude et d'un modèle numérique des cours d'eau et des lacs (TURCOTTE et al., 2001).

Du point de vue de la gestion des bassins versants, GIBSI permet d'élaborer des scénarios d'usage de l'eau et de l'utilisation du territoire à l'aide des modules de gestion suivants :

- ouvrages hydrauliques (ex. : ajout ou retrait d'infrastructures hydrauliques, modification des règles d'opération des infrastructures hydrauliques) ;

- aménagement du territoire (ex. : changements d'occupation du sol, analyse de schémas d'aménagement, etc.);

- rejets ponctuels (ex. : ajout ou retrait de rejets ponctuels urbains et industriels, modifications des caractéristiques de ces rejets);

- pollution diffuse d'origine agricole (ex. : modifications des systèmes de production agricole - cheptel et mode d'épandage des fumiers et lisiers - et des pratiques culturales - fertilisation et protection des cultures, conservation des sols).

À noter que le module de gestion de la pollution diffuse agricole permet de modifier les paramètres de gestion mentionnés précédemment à l'échelle d'une ou plusieurs USS, sous-bassins versants des principaux affluents ou unités administratives (ex. : municipalité). Un outil permettant de sélectionner les USS ayant pour exutoire un tronçon donné permet de grouper une unité de gestion qui ne correspond pas nécessairement à un sous-bassin versant d'un affluent principal. Ainsi, chaque groupe d'entités possède ses propres attributs agricoles. ROUSSEAU et al. (2000a, b) et LAVIGNE et al. (2002) présentent des exemples d'application de GIBSI dans le cadre de l'évaluation des impacts de la pollution diffuse agricole et des rejets municipaux sur la qualité de l'eau ainsi que du déboisement à grande échelle sur le régime hydrique d'un bassin versant.

\subsection{Mise en place du système sur le bassin versant de la rivière Chaudière}

Le bassin versant de la rivière Chaudière (Québec, Canada, voir figure 1) occupe une superficie de $6682 \mathrm{~km}^{2}$ dont $63 \%$ sont en forêt, $17 \%$ en culture et pâturage, $15 \%$ en friche, $3 \%$ en territoire urbanisé et $2 \%$ en eau (VILLE- 
NEUVE et al., 1998b). La population était d'environ 175000 habitants en 1996 (SIMONEAU et al., 1998). Pour la mise en place de GIBSI, le bassin versant a été découpé en 1870 sous-bassins versants élémentaires (USS de superficie moyenne de $\left.3,6 \mathrm{~km}^{2}\right)$ ayant trois couches de sol $(6,25,15,75$ et $178,00 \mathrm{~cm}$ pour les première, deuxième et troisième couches respectivement) et incluant un tronçon de rivière (1 799 tronçons de longueur moyenne de $1,9 \mathrm{~km}$, et 46 tronçons de lacs de longueur moyenne de $1,5 \mathrm{~km}$ ). L'étalonnage du modèle hydrologique de GIBSI est documenté en détail dans FORTIN et al. (2001b). Bien qu'un étalonnage formel de la qualité de l'eau n'ait pas été effectué, un exercice de vérification a permis d'évaluer la cohérence des résultats de la modélisation avec les données observées (VILLENEUVE et al., 1998b, c). Voici les principales conclusions de cette vérification.

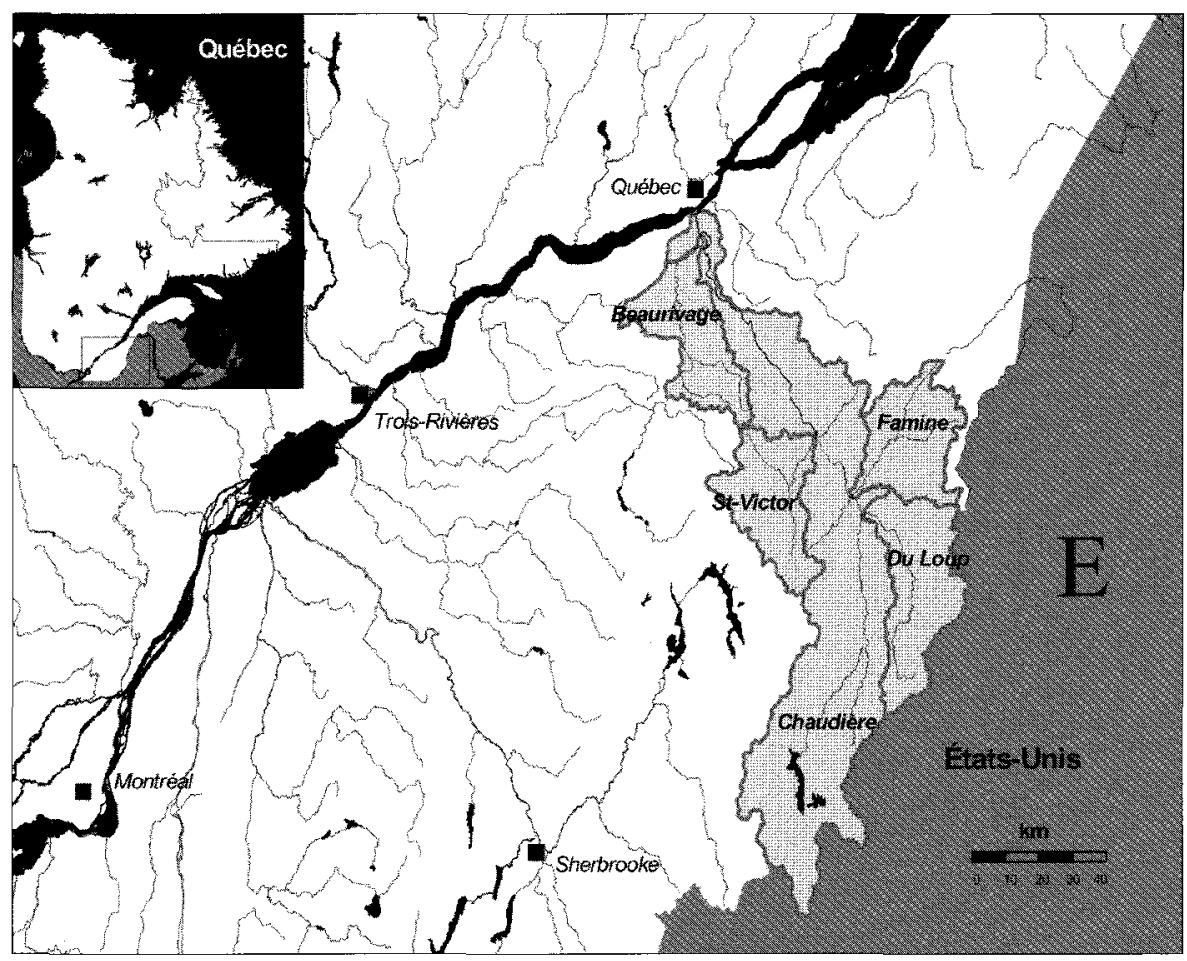

Figure 1 Bassin versant de la rivière Chaudière (Québec, Canada).

Chaudière River Watershed (Québec, Canada).

Cet exercice s'est effectué avec une chronique de données de qualité de l'eau provenant de 12 stations de jaugeage (116 données). L'erreur absolue moyenne entre les valeurs simulées et observées de coliformes fécaux, $C F$, était centrée près de zéro et près de $45 \%$ des écarts étaient inférieurs à $150 \mathrm{UCF} / 100 \mathrm{~mL}$. Ce qui veut dire que $50 \%$ des valeurs observées étaient simulées à $+30 \%$ près. À noter que pour cet exercice, on a supposé, faute de données, une contribution nulle en $C F$ provenant du territoire agricole, des fosses septiques en milieu rural et des débordements de réseaux d'égouts uni- 
taires. Seuls les rejets des stations de traitement des eaux usées municipales contribuaient à cette source de contamination bactériologique. Au niveau du $P$, l'étalonnage s'est effectué avec 108 valeurs observées, dont 56,5\% étaient à $+0,01 \mathrm{mg} P / L$ des valeurs observées - à noter que l'erreur sur les valeurs mesurées est de l'ordre de $+0,005 \mathrm{mg} P / L$. Les écarts les plus importants ont été observés dans les régions agricoles. Ces écarts sont intimement liés aux hypothèses sous-jacentes à l'état de référence du bassin versant de la rivière Chaudière : apports non-répertoriés et différences au niveau des calendriers des pratiques culturales, des règles de fertilisation et des événements météorologiques locaux. II est important de souligner que, pour cet exercice de vérification, on a supposé, faute de données, une contribution nulle en $P$ des fosses septiques en milieu rural et des débordements de réseaux d'égouts unitaires.

\section{4 - CAS DE FIGURE}

Le cas de figure à l'étude correspond à la récupération des activités récréatives, où il y a contact direct et fréquent avec l'eau, sur un tronçon de rivière du bassin versant de la rivière Chaudière, situé en amont d'un ouvrage de retenue, le barrage Sartigan, site de l'usage selon la définition du ministère de l'Environnement du Québec (1991, rév. 2001). Les caractéristiques physiques de ce tronçon sont présentées à la figure 2. Le tableau 1 et la figure 3 donnent le détail de l'aménagement du sous-bassin versant ayant pour exutoire ce tronçon ( $47 \%$ du bassin versant de la rivière Chaudière). Comme on peut le constater, il s'agit d'un sous-bassin versant forestier ( $86 \%$ de la superficie) où l'agriculture se concentre autour des productions laitières et de bovins de boucherie ( $81 \%$ du cheptel) ainsi que des cultures qui les supportent, c'est-à-dire les fourrages et les céréales (67 et $22 \%$ des surfaces cultivées). Les données

Tableau 1 Caractéristiques de l'aménagement du territoire, du cheptel et des surfaces cultivées du bassin versant ayant pour exutoire le tronçon de rivière à l'étude (données de 1996).

Table 1 Land use, livestock and cropland characteristics of the watershed drained by the river segment under study (1996 data).

\begin{tabular}{|c|c|c|c|c|c|c|c|c|c|c|}
\hline \multicolumn{4}{|c|}{$\begin{array}{l}\text { Aménagement du territoire } \\
\qquad\left(\mathrm{km}^{2}\right)\end{array}$} & \multicolumn{4}{|c|}{$\begin{array}{l}\text { Cheptel } \\
\left(\mathrm{UA}^{*}\right)\end{array}$} & \multicolumn{3}{|c|}{$\begin{array}{l}\text { Détail } \\
\text { des surfaces cultivées } \\
\text { (ha) }\end{array}$} \\
\hline 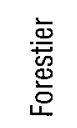 & $\frac{\text { 응 }}{\frac{0}{5}}$ & 旁 & 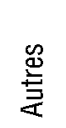 & 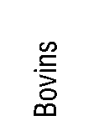 & $\frac{\mathscr{2}}{\mathrm{C}}$ & $\frac{\frac{\mathscr{2}}{5}}{\frac{\bar{\pi}}{5}}$ & 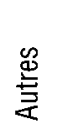 & 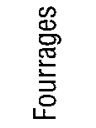 & 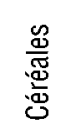 & $\stackrel{\text { : }}{\stackrel{\text { OII }}{\mathbb{N}}}$ \\
\hline 2476 & 218 & 42 & 149 & 12817 & 2104 & 344 & 601 & 14554 & 6940 & 284 \\
\hline
\end{tabular}

* Une unité animale correspond à une consommation de matière sèche annuelle de $6000 \mathrm{~kg}$ ou $16,4 \mathrm{~kg} / \mathrm{jour}$ (i.e., à une vache laitière produisant $6650 \mathrm{~kg}$ de lait/an) (CRÉAC, 1995). 


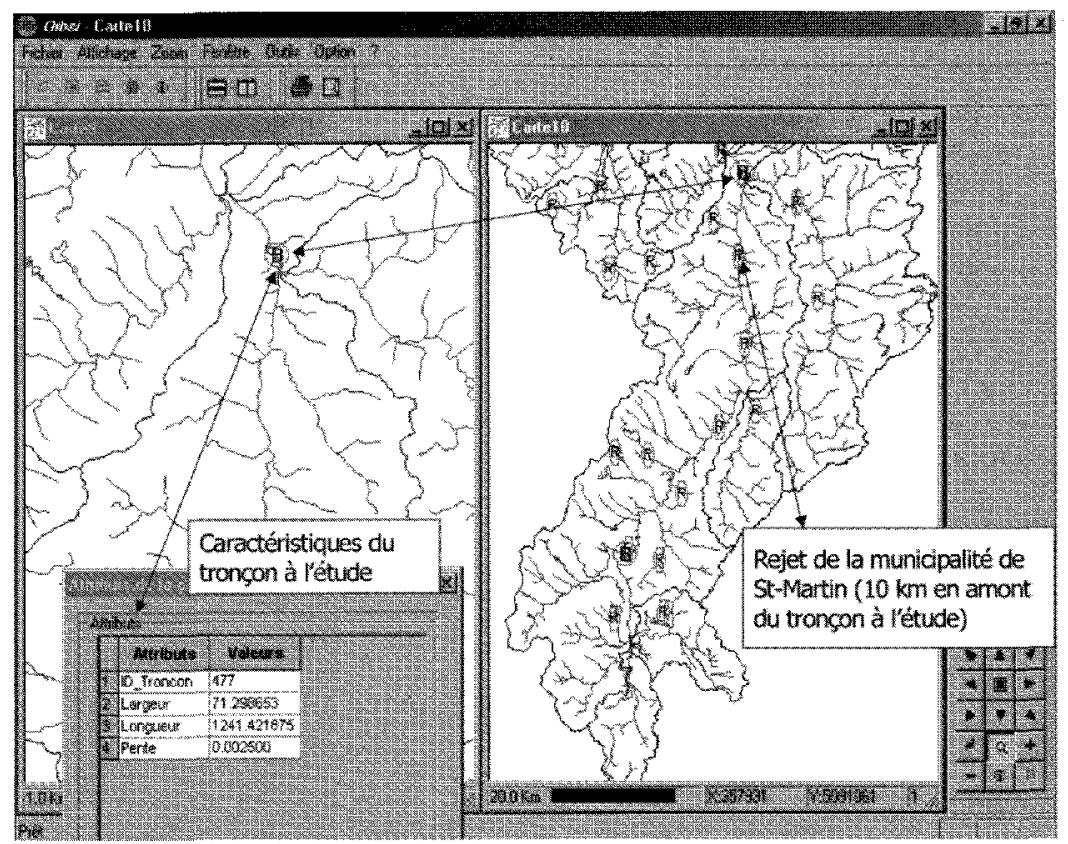

Figure 2 Localisation et caractéristiques du tronçon de rivière à l'étude.

Characteristics and location of the river segment under study.

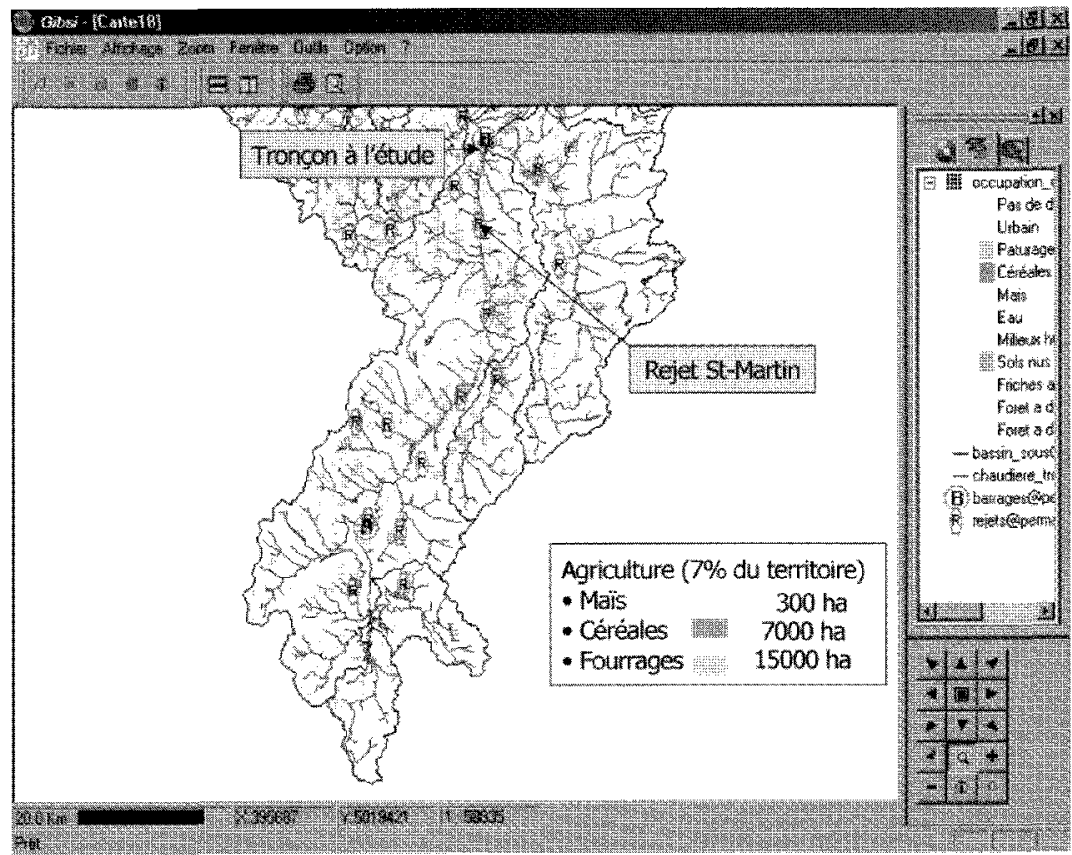

Figure 3 Occupation du sol agricole en amont du tronçon de rivière à l'étude. Agricultural land use upstream of the river segment under study. 
qui caractérisent ce cas de figure proviennent de la base de données qui a servi au développement de GIBSI (VILLENEUVE et al., 1998b).

Les activités de baignade, de planche à voile et de kayak dont il est question requièrent la restauration des propriétés bactériologiques, organoleptiques et esthétiques (ex. : croissance excessive d'algues et autres plantes aquatiques). Pour ce cas de figure, on considère deux critères (Ministère de l'Environnement du Québec, 1990, rév. 1992) : un critère bactériologique pour les $C F, C Q E_{C F}<200 \mathrm{UCF} / 100 \mathrm{~mL}$, et un critère esthétique pour le $P$ total, $C Q E_{P}$ $<0,03 P \mathrm{mg} / \mathrm{L}$ (prévention de l'eutrophisation). La source hypothétique de la contamination bactériologique est le rejet non-traité des eaux usées d'une petite municipalité de 1582 habitants située à $10 \mathrm{~km}$ en amont, la municipalité de St-Martin (voir figures 2 et 3 ). Ce rejet est aussi responsable en partie de l'eutrophisation du plan d'eau tout comme les rejets de $P$ provenant des effluents des stations de traitement des autres municipalités situées en amont. L'autre source majeure de polluant contribuant à l'eutrophisation est d'origine diffuse agricole. À noter que l'on suppose ici des contributions nulles en CF et en $P$ des fosses septiques et des débordements de réseaux d'égouts unitaires, ce qui est justifiable dans les cas où ces concentrations sont négligeables par rapport aux CQE considérés (Ministère de l'Environnement, 1991, rév. 2001).

Tableau 2 Description sommaire des cinq scénarios à l'étude.

Table 2 Short description of the five studied scenarios.

\begin{tabular}{|c|c|c|c|c|}
\hline OER & Scénario & $\begin{array}{c}\text { Sources } \\
\text { de pollution }\end{array}$ & $\begin{array}{c}\text { Eaux usées } \\
\text { de Saint-Martin* }\end{array}$ & $\begin{array}{c}\text { Règles } \\
\text { de fertilisation }\end{array}$ \\
\hline CF & A & Ponctuelle & Non-traitées & Non disponible \\
& B & Ponctuelle & Traitées & Non disponible \\
$P$ & A & Ponctuelle & Non-traitées & Non disponible \\
& B & Ponctuelle & Traitées & Non disponible \\
& C & Ponctuelle et diffuse & Application de \\
& la demande \\
& D & Ponctuelle et diffuse & Non-traitées & Application de l'offre \\
& Ponctuelle et diffuse & Traitées & Application de l'offre \\
\hline
\end{tabular}

${ }^{*}$ On considère dans tous les cas que les eaux usées des municipalités en amont du tronçon à l'étude sont traitées.

\subsection{Construction et simulation de scénarios à l'aide de GIBSI}

\subsubsection{Construction des scénarios}

Cinq scénarios ont été considérés pour l'évaluation des $O E R$ d'origines ponctuelle et diffuse pour les $C F$ et le $P$. Ces scénarios sont définis à partir de deux états de base du bassin versant de la rivière Chaudière (voir tableau 2). Le premier état correspond aux cas où seuls les rejets ponctuels des eaux usées municipales sont considérés et le deuxième état correspond aux cas où les rejets diffus d'origine agricole et les rejets ponctuels d'origine municipale sont considérés simultanément. Nous supposons ici qu'il est possible de découpler 
Tableau 3 Caractéristiques des rejets en $C F$ et $P$ des stations de traitement des eaux usées des municipalités en amont du tronçon à l'étude.

Table $3 \quad F C$ and $\mathrm{P}$ effluent characteristics of the municipal wastewater treatment plants upstream of the river segment under study.

\begin{tabular}{|c|c|c|c|c|c|c|c|}
\hline \multirow{2}{*}{ Municipalité } & \multirow{2}{*}{$\mathbf{N}^{0}$ du rejet $^{* *}$} & \multirow{2}{*}{ Population } & \multirow{2}{*}{$\begin{array}{l}\text { Types de station } \\
\text { et de traitement }\end{array}$} & \multicolumn{2}{|c|}{$C F$} & \multicolumn{2}{|c|}{$P$} \\
\hline & & & & [UCF/100 mL] & [UCF/jour] & {$[\mathrm{mg} / \mathrm{L}]$} & [kg/jour] \\
\hline \multirow[t]{2}{*}{ St-Martin } & \multirow[t]{2}{*}{18} & \multirow[t]{2}{*}{1582} & Aucun & $2 \cdot 10^{6}$ & $2,3 \cdot 10^{13}$ & 2,74 & 3,16 \\
\hline & & & $E A$ avec $D P$ & $2 \cdot 10^{3}$ & $2,3 \cdot 10^{10}$ & 1,01 & 1,17 \\
\hline St-Côme-de-Kennebec & 22 & 1978 & $E A$ avec $D P$ & $2 \cdot 10^{3}$ & $2,9 \cdot 10^{10}$ & 1,01 & 1,46 \\
\hline St-Jean-de-La-Lande & 19 & 354 & $E A$ & $2 \cdot 10^{3}$ & $5,2 \cdot 10^{9}$ & 1,01 & 0,26 \\
\hline St-Théophile & 21 & 438 & $E A$ & $2 \cdot 10^{3}$ & $6,4 \cdot 10^{9}$ & 1,01 & 0,32 \\
\hline St-Gédéon & 16 & 1538 & $E A$ & $2 \cdot 10^{3}$ & $2,2 \cdot 10^{10}$ & 1,01 & 1,13 \\
\hline Risborough & 14 & 654 & $E A$ avec $D P$ & $2 \cdot 10^{3}$ & $9,5 \cdot 10^{9}$ & 1,01 & 0,48 \\
\hline St-Robert-Bellarmin & 15 & 353 & ENA & $2 \cdot 10^{3}$ & $5,2 \cdot 10^{9}$ & 2,05 & 0,53 \\
\hline Lac Drolet & 11 & 255 & Aucun & $2 \cdot 10^{6}$ & $3,7 \cdot 10^{12}$ & 2,74 & 0,51 \\
\hline Audet & 10 & 285 & $F S$ & 0 & 0 & 0 & 0 \\
\hline Ste-Cécile-de-Whitton (St-Samuel) & 7 & 356 & Aucun & $2 \cdot 10^{6}$ & $5,2 \cdot 10^{12}$ & 2,74 & 0,71 \\
\hline Lac Mégantic & 5 & 6195 & $B A$ avec $D P$ & $2 \cdot 10^{3}$ & $9,0 \cdot 10^{10}$ & 0,47 & 2,13 \\
\hline Frontenac Village & 9 & 305 & ENA & $2 \cdot 10^{3}$ & $4,5 \cdot 10^{9}$ & 2,05 & 0,46 \\
\hline Piopolis & 2 & 280 & FS & 0 & 0 & 0 & 0 \\
\hline Frontenac (Mercier) & 8 & 125 & $E N A$ avec $D P$ & $2 \cdot 10^{3}$ & $1,8 \cdot 10^{9}$ & 2,05 & 0,19 \\
\hline
\end{tabular}

*Débit moyen annuel du rejet : $0,73 \mathrm{~m}^{3} /$ personne/j; voir figure 6 pour localisation géographique du rejet des municipalités.

${ }^{* \star} E A$ : étangs aérées ; $E N A$ : étangs non aérés ; $B A$ : boues activées ; $F S$ : fosses septiques ; $D P$ : déphosphatation. 
les influences des rejets ponctuels sur les rejets diffus, et vice versa, sur la qualité de l'eau. Ceci équivaut à stipuler que les phénomènes non linéaires sont négligeables et que seuls les phénomènes additifs sont susceptibles de se produire en ce qui concerne les CF et le $P$ des différents rejets. Chacun de ces deux états de base est ensuite caractérisé selon le non-traitement (scénarios $A$, $C$ et D) ou le traitement des eaux usées municipales de la municipalité de StMartin (scénarios B et E). Lorsqu'il y a traitement, on suppose que celui-ci correspond à la mise en opération d'étangs aérés (avec déphosphatation). Pour les autres municipalités en amont du plan d'eau à l'étude, on considère dans tous les cas la situation actuelle (voir tableau 3). Le type de traitement a été identifié lors de la construction de la base de données ayant servi au développement de GIBSI (VILLENEUVE et al., 1998b).

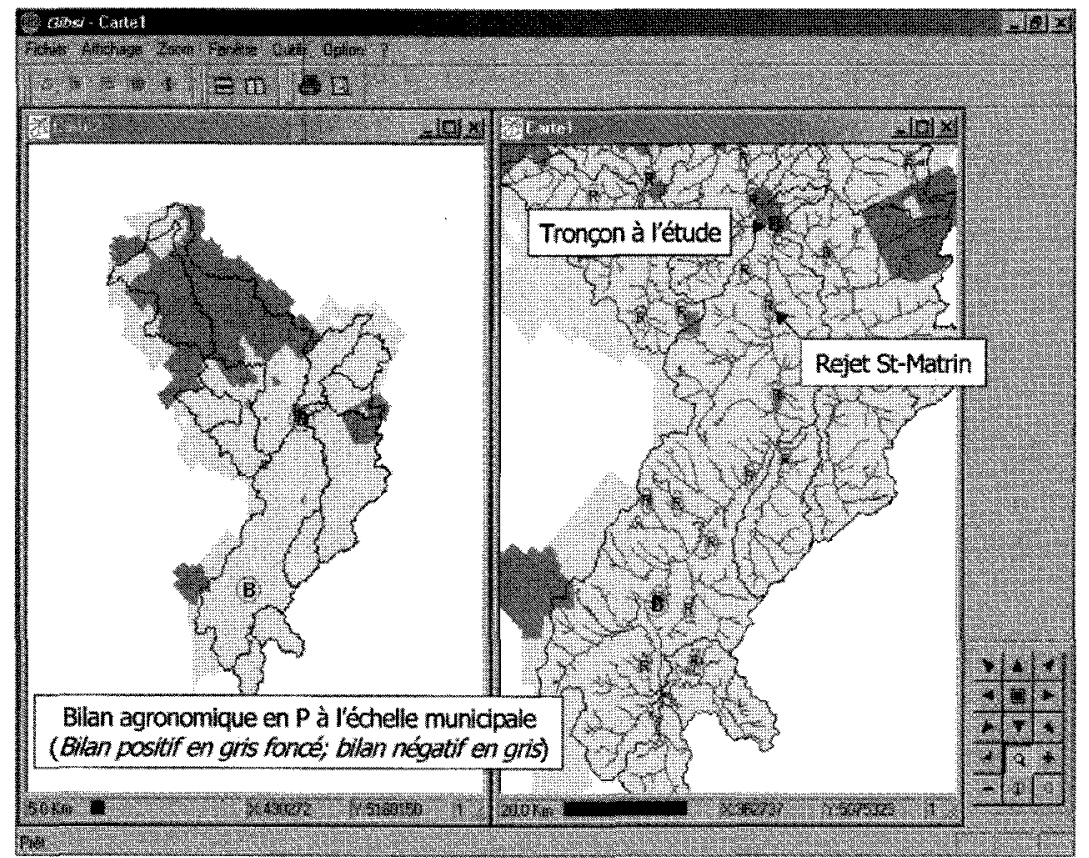

Figure 4 Bilans agronomiques, à l'échelle de la municipalité, du bassin versant de la rivière Chaudière (1996).

Chaudière River Watershed municipal agronomic mass balances (1996).

Lorsque les rejets diffus sont inclus, deux règles de fertilisation des sols cultivés sont considérées. La première règle correspond à la satisfaction des demandes agronomiques en $N$ et $P$ (scénario $C$ ) et la deuxième à l'application des engrais de ferme disponibles (i.e., fumiers et lisiers produits par le cheptel), sans égard aux demandes agronomiques (scénarios $D$ et $E$ ). Étant donné que les bilans agronomiques pour le bassin versant alimentant ce tronçon sont négatifs $(-30 \mathrm{~kg} \mathrm{~N} / \mathrm{ha}$ et $-18 \mathrm{~kg} P / \mathrm{ha}$, basés sur l'offre en engrais de ferme disponible et de la demande agronomique des cultures), le scénario $\mathrm{C}$ produira une plus grande pression agricole sur le bassin versant $(52 \mathrm{~kg} \mathrm{~N} / \mathrm{ha}$ et $31 \mathrm{~kg}$ 
$P /$ ha) que les scénarios $D$ et $E(22 \mathrm{~kg} \mathrm{~N} / \mathrm{ha}$ et $13 \mathrm{~kg} P / \mathrm{ha})$. Ces taux de fertilisation ont été calculés à l'aide des données sur les attributs agricoles du bassin versant de la rivière Chaudière, incluses dans la base de données de GIBSI, et des données rapportées dans les grilles de référence en fertilisation du CPVQ (1996). La figure 4 présente la distribution des bilans agronomiques positifs et négatifs pour chacune des municipalités du bassin versant de la rivière Chaudière (calculés par GIBSI à partir du cheptel et des cultures inventoriés lors du développement de ce système). Les pratiques de fertilisation pour les principales cultures (i.e., fourrages, céréales et maïs) se déroulent principalement avant la période estivale (mois de mai) sauf pour le maïs où il y a une fertilisation supplémentaire au stade de post-levée (CPVQ, 1996).

\subsubsection{Chroniques météorologiques et débits critiques}

La simulation de ces scénarios requiert la sélection de chroniques météorologiques annuelles incluant au moins une chronique produisant un débit critique correspondant au débit d'étiage de 30 jours d'une période de retour de 5 ans $\left(Q_{5-30}\right)$ associé au tronçon de rivière à l'étude (voir note de bas de page numéro 3). Les chroniques météorologiques utilisées pour ce cas de figure sont celles des chroniques 1982-19854 où l'année 1982 est caractérisée par un $Q_{5-30}$ estival à la station hydrométrique la plus près de l'exutoire du bassin, soit la station de St-Lambert. Cette caractérisation a été faite à partir d'une analyse statistique des données enregistrées à cette station (VILLENEUVE et al., 1998b). On suppose que la chronique 1982 a également produit un tel débit d'étiage sur les tronçons de rivière considérés ici, c'est-à-dire, le tronçon dans lequel le rejet des eaux usées de la municipalité de St-Martin se déverse et celui pour lequel on entend restaurer les usages récréatifs, le tronçon en amont du barrage Sartigan. Comme il n'y a pas de station de jaugeage sur ces tronçons, on a supposé que le $Q_{5-30}$ estival associé à ces derniers se produisait durant la même période que celle ayant produit le $Q_{5-30}$ estival à la station de St-Lambert (i.e., 10 juillet au 8 août). Quant à la valeur de ces débits d'étiage, on l'a estimée à partir des résultats de simulation de GIBSI. Ainsi, la valeur du $Q_{5-30}$ estival pour le tronçon recevant le rejet des eaux usées de la municipalité de St-Martin est estimée à $4,32 \mathrm{~m}^{3} / \mathrm{s}$ et celle pour le tronçon en amont du barrage Sartigan est estimée à $6,05 \mathrm{~m}^{3} / \mathrm{s}$. La figure 5 présente les chroniques de débits simulés au tronçon de rivière en amont du barrage Sartigan pour la période estivale des chroniques 1982 à 1985 . Pour ces chroniques-là, les débits sur le tronçon ont été respectivement inférieurs au $Q_{5-30}$ estival 31, 62, 19 et 2 jours au cours de la période estivále (i.e., 21 juin au 20 septembre). Ce qui est particulier, c'est le nombre de jours où le débit est inférieur au $Q_{5-30}$ en 1983 par rapport à l'année 1982.

\subsubsection{Caractéristiques des rejets des eaux usées et approximation d'OER}

Les caractéristiques du rejet des eaux usées de la municipalité de St-Martin, avec ou sans traitement, ont été présentées au tableau 3. Ce tableau présente également les caractéristiques des rejets des eaux usées des autres municipalités situées en amont du tronçon d'eau à restaurer (13 rejets, voir

4. Pour des raisons pratiques, on a choisi une chronique s'étalonnant sur quatre ans. Idéalement, on devrait avoir recours à de longues chroniques météorologiques (ex. : 30 ans). 


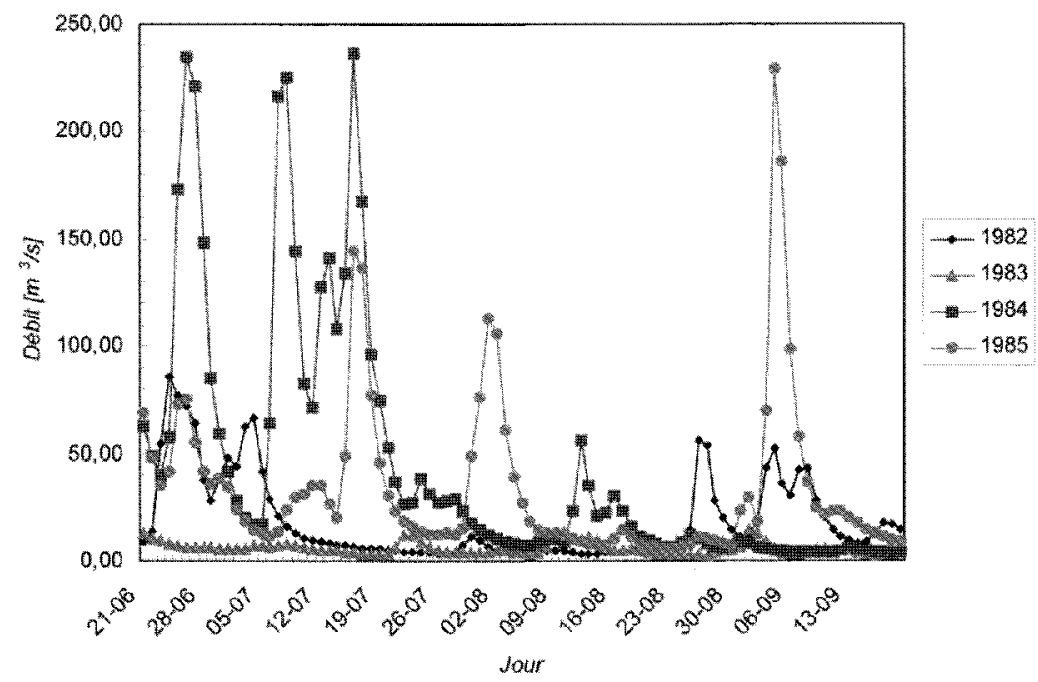

Figure 5 Débits simulés au tronçon de rivière à l'étude pour la période estivale des chroniques 1982 à 1985.

Simulated summer stream flows at the studied river segment (1982 through 1985).

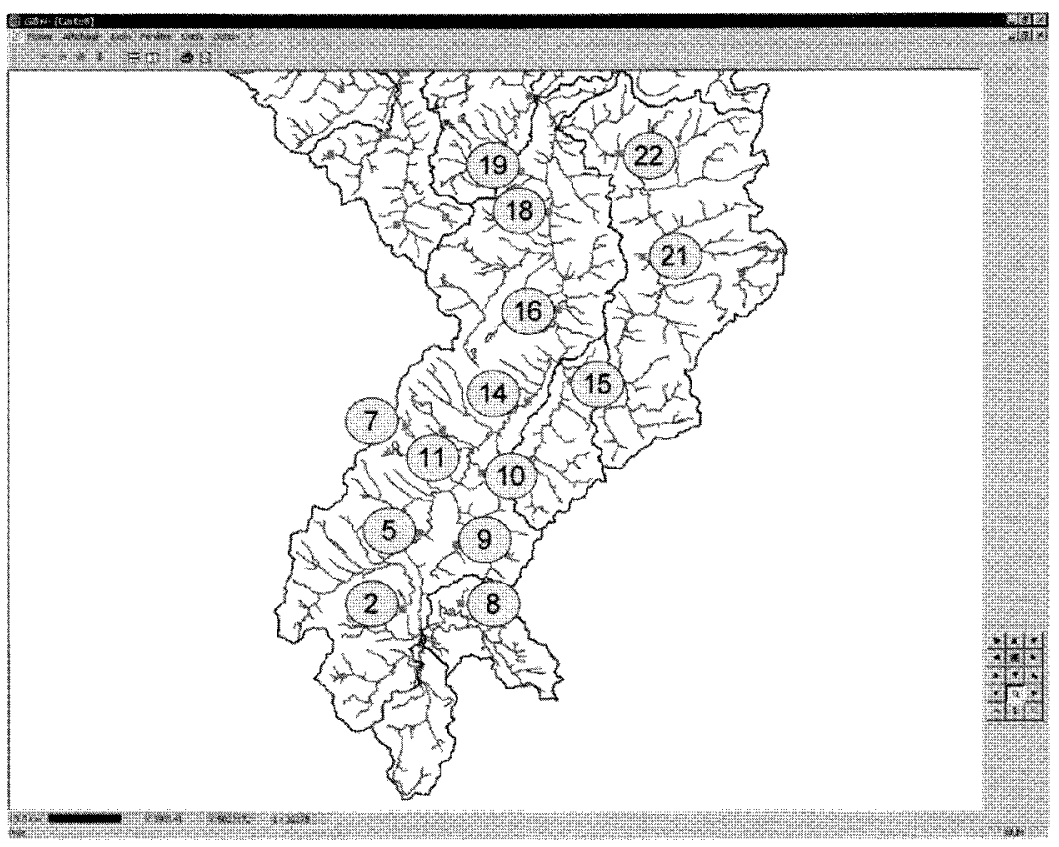

Figure 6 Localisation de tous les rejets municipaux en amont du tronçon à l'étude (voir tableau 3 donnant la liste des noms des municipalités).

Location of all municipal discharges upstream of the river segment under study (see table 3 for link between discharge number and name of municipality). 
figure 6). Les charges rejetées au cours d'eau en $C F$ et en $P$ provenant du rejet de la municipalité de St-Martin sont de $2,3 \cdot 10^{13} \mathrm{UCF} / \mathrm{jour}$ et de $3,16 P \mathrm{~kg} / \mathrm{jour}$ lorsqu'il y a aucun traitement et de $2,3 \cdot 10^{10} \mathrm{UCF} / \mathrm{jour}$ et de $1,17 \mathrm{P} \mathrm{kg} / \mathrm{jour}$ lorsqu'il y a traitement.

En supposant que les charges provenant des stations de traitement en amont (incluant le non-traitement des eaux usées de St-Martin) soient négligeables pour les CF (i.e., polluant « non conservatif » sujet au processus de dégradation) et de $11,33 \mathrm{~kg} / \mathrm{jour}$ pour le $P$ (i.e., polluant " conservatif "), on estime qu'en période d'étiage $Q_{5-30}$, les $O E R$, incluant les contributions d'origines diffuse et ponctuelle, sont respectivement de $7,5 \cdot 10^{11}$ UCF/jour et de $4,35 \mathrm{~kg} P /$ jour pour le tronçon à restaurer. Si on traitait avec des étangs aérés (avec déphosphatation) le rejet d'eaux usées de St-Martin, l'OER pour le $P$ deviendrait $6,34 \mathrm{~kg} P / \mathrm{j}$. Dans les deux cas, si l'on entend restaurer les usages récréatifs du tronçon en amont du barrage Sartigan, les rejets diffus d'origine agricole en amont ne devraient pas être supérieurs à 4,35 ou $6,34 \mathrm{~kg} P /$ jour en période d'étiage $Q_{5-30}$ selon le cas (i.e., pour les CF et $P, C H A>O E R_{\text {ponctuelle }}+$ $O E R_{\text {Diffuse }}$, voir équation $\left.4 b\right)$.

Tableau 4 Probabilités de dépassement pour la période estivale des critères bactériologique (CQE $C$ CF $200 \mathrm{UCF} / 100 \mathrm{~mL}$ ) et esthétique (CQE $<0,03 P \mathrm{mg} / \mathrm{L}$ ) pour les cinq scénarios.

Table 4 Probability of exceeding bacteriological $\left(W Q S_{F C}<200\right.$ UFC/100 mL) and aesthetic $\left(W Q S_{P}<0,03 P \mathrm{mg} / \mathrm{L}\right)$ standards during the summer period for the five studied scenarios.

\begin{tabular}{|c|c|c|c|c|c|c|c|c|c|c|}
\hline \multirow{2}{*}{ Scénario } & \multicolumn{5}{|c|}{$\mathrm{P}\left([C F]>C Q E_{C F}\right)$} & \multicolumn{5}{|c|}{$\mathrm{P}\left([P]>C Q E_{p}\right)$} \\
\hline & 82 & 83 & 84 & 85 & $82-85$ & 82 & 83 & 84 & 85 & $82-85$ \\
\hline A & 1 & 1 & 0,84 & 0,94 & 0,94 & 0,16 & 0,33 & 0,10 & 0 & 0,14 \\
\hline$B$ & 0 & 0 & 0 & 0 & 0 & 0,04 & 0,17 & 0,02 & 0 & 0,05 \\
\hline C & 1 & 1 & 0,84 & 0,94 & 0,94 & 0,36 & 0,52 & 0,28 & 0,20 & 0,32 \\
\hline D & 1 & 1 & 0,84 & 0,94 & 0,94 & 0,33 & 0,46 & 0,22 & 0,11 & 0,27 \\
\hline$E$ & 0 & 0 & 0 & 0 & 0 & 0,22 & 0,33 & 0,17 & 0,09 & 0,19 \\
\hline
\end{tabular}

\section{5 - PRÉSENTATION, ANALYSE ET DISCUSSION DES RÉSULTATS}

Le tableau 4 présente, pour les cinq scénarios simulés à l'aide de GIBSI et pour la période estivale (21 juin au 20 septembre) des chroniques 1982 à 1985, les probabilités de dépassement des critères bactériologique ( $C Q E_{C F}$ $<200 \mathrm{UCF} / 100 \mathrm{~mL})$ et esthétique $\left(C Q E_{P}<0,03 P \mathrm{mg} / \mathrm{L}\right)$ sur le tronçon associé au plan d'eau à restaurer. Le tableau 5 introduit les charges totales de $C F$ et de $P$ passant par ce tronçon durant la saison estivale des chroniques 1982 à 1985 et le tableau 6 les proportions d'origines diffuse et ponctuelle des charges de $P$. Finalement, le tableau 7 présente l'écoulement estival total et les charges maximales totales de $C F$ et de $P$ passant par le tronçon associé au plan d'eau 
Tableau 5 Charges totales de $C F$ et de $P$ passant par le tronçon à l'étude durant la saison estivale des chroniques 1982 à 1985.

Table 5 Total loads of FC and $P$ for the study river segment for the summer season (1982 through 1985).

\begin{tabular}{|c|c|c|c|c|c|c|c|c|}
\hline \multirow{2}{*}{ Scénario } & \multicolumn{4}{|c|}{$\begin{array}{c}\text { Charges de CF } \\
\left(\mathbf{1 0 ^ { 1 4 }} \text { UCF) }\right.\end{array}$} & \multicolumn{4}{|c|}{$\begin{array}{c}\text { Charges de } \boldsymbol{P} \\
\text { (t) }\end{array}$} \\
\cline { 2 - 9 } & $\mathbf{8 2}$ & $\mathbf{8 3}$ & $\mathbf{8 4}$ & $\mathbf{8 5}$ & $\mathbf{8 2}$ & $\mathbf{8 3}$ & $\mathbf{8 4}$ & $\mathbf{8 5}$ \\
\hline A & 14 & 11,3 & 13,8 & 15,3 & 1,06 & 1,03 & 1,02 & 1,06 \\
B & 0,8 & 0,3 & 0,7 & 1 & 0,89 & 0,86 & 0,85 & 0,89 \\
C & 14 & 11,3 & 13,8 & 15,3 & 3,66 & 1,63 & 8,4 & 4,89 \\
D & 14 & 11,3 & 13,8 & 15,3 & 2,85 & 1,39 & 5,98 & 3,84 \\
E & 0,8 & 0,3 & 0,7 & 1 & 2,68 & 1,22 & 5,81 & 3,67 \\
\hline
\end{tabular}

Tableau 6 Proportions des charges en $P$ diffuses et ponctuelles passant par le tronçon à l'étude durant la saison estivale des chroniques 1982 à 1985.

Table 6 Contributions of nonpoint and point source $P$ loads for the summer season (1982 through 1985).

\begin{tabular}{|c|c|c|c|c|c|c|c|c|}
\hline \multirow{2}{*}{ Scénario } & \multicolumn{8}{c|}{ Contributions des charges en P(\%) } \\
\cline { 2 - 10 } & \multicolumn{2}{|c|}{$\mathbf{8 2}$} & \multicolumn{2}{|c|}{$\mathbf{8 3}$} & \multicolumn{2}{c|}{$\mathbf{8 4}$} & \multicolumn{2}{c|}{$\mathbf{8 5}$} \\
\cline { 2 - 10 } & $\mathbf{P}^{*}$ & $\mathbf{D}^{* *}$ & $\mathbf{P}$ & $\mathbf{D}$ & $\mathbf{P}$ & $\mathbf{D}$ & $\mathbf{P}$ & $\mathbf{D}$ \\
\hline \multirow{2}{*}{ A } & 100 & 0 & 100 & 0 & 100 & 0 & 100 & 0 \\
B & 100 & 0 & 100 & 0 & 100 & 0 & 100 & 0 \\
C & 29 & 71 & 63 & 37 & 12 & 88 & 22 & 78 \\
D & 40 & 60 & 74 & 26 & 17 & 83 & 28 & 72 \\
E & 33 & 67 & 70 & 30 & 14 & 86 & 24 & 76 \\
\hline
\end{tabular}

${ }^{\star}$ Ponctuelles; ${ }^{\star *}$ Diffuses

Tableau 7 Écoulement total et charges maximales totales de CF et de $P$ passant par le tronçon à l'étude durant la saison estivale des chroniques 1982 à 1985 .

Table 7 Total runoff volume, nonpoint and point maximum $P$ loads for the summer season (1982 through 1985).

\begin{tabular}{|c|c|c|c|}
\hline Chronique & $\begin{array}{c}\text { Écoulement total } \\
\left(\mathbf{( 1 0}^{\mathbf{6}} \mathbf{~}^{\mathbf{3}} \mathbf{)}\right.\end{array}$ & $\begin{array}{c}\text { Charge maximale } \mathbf{C F} \\
\left(\mathbf{1 0}^{\mathbf{1 4}} \mathbf{U C F}\right)\end{array}$ & $\begin{array}{c}\text { Charge maximale } \boldsymbol{P} \\
\mathbf{( t )}\end{array}$ \\
\hline 1982 & 150 & 3 & 4,5 \\
1983 & 48 & 1 & 1,43 \\
1984 & 348 & 7 & 10,44 \\
1985 & 270 & 5,4 & 8,09 \\
\hline
\end{tabular}


à restaurer, et cela pour chacune des années simulées. Ces charges maximales sont égales au produit du débit simulé et du CQE correspondant.

\subsection{OER d'origine ponctuelle pour les $C F$ et le $P$ (scénarios $A$ et $B$ )}

Les résultats des scénarios $A$ et $B$ montrent que la mise en place d'étangs aérés (avec déphosphatation) pour le traitement des eaux usées de la municipalité de St-Martin permet d'abaisser à zéro la probabilité de dépassement du critère bactériologique (réduction de la charge en $C F$ variant entre 93 et $97 \%$ ) (voir tableau 4 et pour un exemple de courbes de probabilité, voir figure 7). À noter que lorsqu'il n'y avait pas de traitement des eaux usées, ce critère n'était pas toujours dépassé durant la période estivale des chroniques 1984 et 1985 $\left\{\mathrm{P}\left([\mathrm{CF}]>C Q E_{C F}\right)=0,84\right.$ et 0,94$\}$, chroniques caractérisées par des écoulements estivaux totaux importants $\left(348 \cdot 10^{6}\right.$ et $\left.270 \cdot 10^{6} \mathrm{~m}^{3}\right)$ par rapport à ceux des chroniques 1982 et $1983\left(150 \cdot 10^{6}\right.$ et $48 \cdot 10^{6} \mathrm{~m}^{3}$, voir tableau 7$)$. D'un point de vue d'OER d'origine ponctuelle, les résultats de simulation du scénario $\mathrm{B}$ montrent que la charge totale de CF passant par le plan d'eau à restaurer est toujours inférieure à la charge maximale totale (telle que définie à l'équation 1a) pour la période estivale $\left(0,3 \cdot 10^{14}\right.$ UCF $<$ charge totale de $C F<1,0 \cdot 10^{14}$ UCF toujours inférieure à $1,0 \cdot 10^{14}$ UCF $<$ charge maximale totale $C F<7,0 \cdot 10^{14}$ UCF, voir tableaux 5 et 7 ).

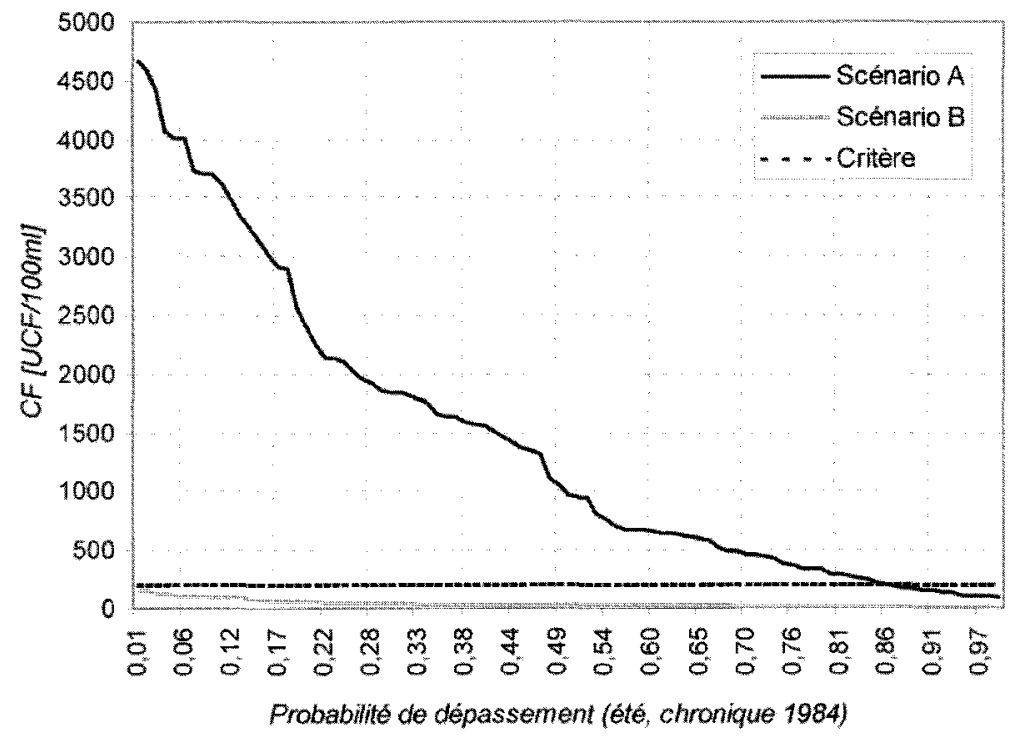

Figure 7 Courbes de probabilités de dépassement du critère bactériologique $\left(C Q E_{C F}<200 \mathrm{UCF} / 100 \mathrm{~mL}\right)$ pour les scénarios A et B pour la chronique 1984.

1984 probability curve of exceeding the bacteriological water quality standard $\left(\mathrm{WQS}_{\mathrm{FC}}<200\right.$ UFC/100 $\mathrm{mL}$ ) for scenarios $A$ and $B$ and for 1984. 
Quant au critère esthétique, la probabilité de dépassement de ce dernier a été globalement (échantillon 1982-1985) réduite de 0,14 à 0,05 (réduction de la charge en $P$ variant entre 16 et $17 \%$ ) lorsqu'il y a traitement des eaux usées (scénario B). À noter que la probabilité de dépassement était de zéro pour les chroniques, 1982, 1984 et 1985 mais qu'elle n'avait en revanche pas été réduite à zéro pour la chronique 1983, même si la charge maximale totale pour la période estivale n'avait pas été dépassée $(0,85 t<$ charge totale de $P$ $<0,89 \mathrm{t}$ toujours inférieure à $1,43 \mathrm{t}<$ charge maximale totale $P<10,44 \mathrm{t}$, voir tableaux 5 et 7 ). Ceci sous-entend que s'il faut réduire cette probabilité, il faudra mettre en place un traitement supplémentaire afin de réduire la charge en $P$ du rejet de la municipalité ou simuler un scénario où l'on imposerait un traitement plus sévère à une autre station de traitement jugée responsable de la dégradation des eaux (ex. : St-Jean-de-La-Lande, voir figure 6 et tableau 3).

Dans un autre ordre d'idée, on a estimé au paragraphe 4.1.3 que l'OER pour tout le $P$ émis en amont du tronçon à l'étude était de 6,34 ou $4,35 \mathrm{~kg} P$ /jour (débit d'étiage critique de $6,05 \mathrm{~m}^{3} / \mathrm{s}$ ) si l'on traitait ou pas le rejet de StMartin. Étant donné que les rejets ponctuels sont constants dans le temps et que les $O E R$ d'origine ponctuelle sont établis à partir d'un débit critique, cela suggère que le débit dans le tronçon a été globalement (échantillon 1982-1985) inférieur à ce débit critique 5 jours (scénario B) et 13 jours (scénario A) au cours de la période estivale. Ce qui fait ressortir l'importance d'établir des OER sur une base de probabilités de dépassement journalières d'un CQE plutôt que sur la base d'un débit critique moyen auquel on rattache une période de retour annuelle, ou encore sur la base d'une charge souhaitable intégrée sur une longue période de temps comme on a tendance à le faire pour le contrôle de la pollution diffuse d'origine agricole (ex. : charges de sédiments liées à l'érosion des sols).

\subsection{OER d'origine diffuse pour le $P$ (scénarios $C$ et $D$ )}

Le scénario $C$ représente le point de départ. Les eaux usées de la municipalité de St-Martin ne sont pas traitées et la pression agricole sur le bassin versant découle de l'application des demandes agronomiques sur les surfaces cultivées, où la pression était la plus forte $(31 \mathrm{~kg} P /$ ha). Pour ce scénario, la probabilité de dépassement du critère esthétique pour les chroniques 1982 à 1985 est de 0,32. La probabilité maximale était de 0,52 pour la chronique 1983, une chronique caractérisée par un écoulement estival total très faible par rapport aux autres chroniques simulées (voir tableau 7). En 1983, la contribution d'origine ponctuelle à la charge totale en $P$ était de $63 \%$, alors que celle d'origine diffuse était de $37 \%$ (voir tableau 6). Pour toutes les autres chroniques, la contribution d'origine diffuse dominait (entre 71 et $88 \%$ ).

Les résultats de la simulation du scénario $D$ illustrent comment on peut réduire cette pression d'origine agricole en appliquant l'offre en $P$ (13 kg $P /$ ha). Pour ce scénario, la probabilité de dépassement du critère esthétique pour les chroniques 1982 à 1985 passe de 0,32 à 0,27 pour un gain moyen de cinq jours ( 25 jours au lieu de 30 jours de dépassement) et un gain maximal de huit jours pour la chronique 1985 (10 jours au lieu de 18 jours de dépassement). Pour cette dernière chronique, on a observé une réduction de $1,05 \mathrm{t}$ de la charge totale en $P$, ce qui équivaut à une réduction de $27 \%$ de la pollution diffuse d'origine agricole (voir tableau 5). 


\subsection{OER d'origines ponctuelle et diffuse pour le $P$ (scénarios $\mathrm{C}, \mathrm{D}$ et $\mathrm{E}$ )}

Les résultats de simulation des scénarios $C$ et $E$ illustrent comment les probabilités de dépassement des critères esthétique et bactériologique sont globalement passées de 0,32 à 0,19 et de 0,94 à 0 en réduisant les pressions agricole et urbaine (réduction de la charge en $P$ variant entre 16 et $17 \%$ pour le milieu urbain et entre 31 et $40 \%$ pour le milieu agricole). Au niveau du critère esthétique associé au rejet de $P$, si l'on compare les résultats des scénarios $D$ et $\mathrm{E}$, on se rend compte que l'assainissement urbain de la municipalité de StMartin a permis de réduire cette probabilité de 0,27 à 0,19. De même, si l'on compare les résultats des scénarios $\mathrm{E}$ et $\mathrm{B}$, la contribution de la pollution diffuse découlant de la pression agricole a fait passer la probabilité de dépassement de 0,05 à 0,19 , par rapport à la contribution de la pollution ponctuelle, soit une contribution de 13 jours de dépassement sur un total de 18 jours de dépassement pour la période estivale. Ceci se reflète surtout au niveau des chroniques de 1982, 1983 et 1985 où la contribution d'origine diffuse représentait 67,86 et $76 \%$ de la charge totale de $P$. En revanche, pour la saison estivale " sèche " de la chronique 1983, ce constat est inversé, la contribution d'origine ponctuelle représentant $70 \%$ de la charge totale de $P$. Ainsi, pour cette période estivale, la pollution d'origine ponctuelle a été responsable du dépassement du critère esthétique pendant 16 jours sur une possibilité de 30 jours, soit un peu plus de $50 \%$ du temps.

\section{6 - CONCLUSION}

La présente étude a permis d'illustrer, à partir d'un cas de figure simulé avec le système de modélisation intégrée GIBSI de VILLENEUVE et al. (1998b), un nouveau concept d'application d'objectifs environnementaux de rejet (OER) d'origine ponctuelle ou diffuse, basé sur l'évaluation d'une probabilité de dépassement. Plus particulièrement, on a montré, dans le cadre d'une analyse de scénarios de restauration des activités récréatives à contact direct et fréquent avec l'eau sur le bassin versant de la rivière Chaudière (Québec, Canada), l'importance de bien départager les interventions urbaines des interventions agricoles. Par exemple, les résultats de simulation à l'aide de quelques chroniques météorologiques ont permis d'illustrer comment les probabilités de dépassement des critères esthétique et bactériologique sont globalement passées de 0,32 à 0,19 et de 0,94 à 0 en réduisant en moyenne les pressions agricole et urbaine de 32 et $17 \%$ respectivement.

À noter que l'on n'a pas cherché à déterminer, sur une base journalière, si la cause du dépassement était liée plus à un rejet ponctuel qu'aux rejets diffus, ou encore si ce dépassement était dû à un faible débit ou à une charge importante bien que la modélisation le permette. On n'a pas non plus cherché à explorer l'impact de scénarios plus ciblés tant du point de vue spatial que temporel même si GIBSI offre ces possibilités (ex. : un scénario de réduction de la pression agricole ne touchant que la portion aval du bassin versant au lieu de la totalité du bassin versant). 
Ce qu'il faut retenir ici, c'est l'interprétation des $O E R$ en termes de probabilités de dépassement de CQE. Dans le cadre d'un rejet d'origine ponctuelle où l'on utilise un débit de design pour calculer un OER et son risque de dépassement, il faut bien retenir qu'il y aura des violations des $C Q E$ à l'étude même si l'on se retrouve dans les conditions ayant conduit à ce débit car, par définition, il y aura des journées incluses dans la durée d'étiage choisie où le débit sera inférieur au débit de design. D'où l'importance de rapporter le risque en matière de dépassement d'un CQE pour la période de contrôle plutôt que sur la base du dépassement d'un débit de design d'une période de retour donnée. Pour ce qui est de la pollution diffuse, il s'avère également plus intuitif de caractériser des scénarios d'interventions d'atteinte d'un OER sur une base de probabilités de dépassement de CQE à l'aide de simulations hydrologiques basées sur de longues chroniques météorologiques. Ainsi, la méthodologie proposée permet de quantifier les risques associés à l'implantation d'interventions en matière du nombre de jours moyen où le CQE est dépassé durant la période de contrôle. Pour le gestionnaire, cette façon de faire permet de communiquer aux décideurs un risque plus exact, et surtout, basé sur ce qui est mesuré en rivière, c'est-à-dire les concentrations de polluants. À noter que pour certains paramètres de qualité de l'eau, il faut de plus apporter un jugement sur la détérioration esthétique des plans d'eau (propriétés visuelles et olfactives) ce que la modélisation ne permet pas en ce moment. Cette vérification se fait par une visite sur les lieux où l'on examine la présence de nuisances (ex. : prolifération de plantes en suspension ou enracinées).

\section{7 - TRAVAUX FUTURS}

Il existe plusieurs méthodes pour quantifier l'incertitude ou le risque associé à l'implantation d'un programme de contrôle de la pollution diffuse élaborée à partir de travaux de simulation hydrologique (quantité et qualité de l'eau). On pense ici aux analyses d'incertitudes telles que celles produites à partir de simulations basées sur:

- l'échantillonnage Monte Carlo des valeurs possibles des principaux paramètres des modèles déterministes [voir par exemple les travaux de HESSION et STORM (2000) ou YULIANTI et al. (1999)] ;

- ou encore l'échantillonnage stratifié issu de la technique Jacknife sur la réalisation conjointe de plusieurs facteurs de réduction des charges ponctuelles et diffuses [voir les travaux de CHEN et al. (1998)]. L'utilisation de ces analyses est complémentaire aux travaux présentés dans cette communication. Des travaux futurs viseront à intégrer ces techniques au calcul de probabilité de dépassement d'OER d'origines ponctuelle et diffuse, ceci dans le but de fournir au gestionnaire un outil d'aide à la décision qui inclut tant les incertitudes reliées aux aléas météorologiques que les incertitudes associées aux valeurs des paramètres des modèles.

II existe également un besoin de combiner à ces travaux le développement d'une méthodologie d'analyse coûts-avantages de scénarios de contrôle. Ceci est d'autant plus important lorsque l'on considère les investissements publics 
et privés que pourrait encourir tout programme d'assainissement des eaux. Des travaux dans cet axe de recherche sont déjà en cours et feront l'objet de communications dans un avenir rapproché.

\section{REMERCIEMENTS}

Nous remercions MM. Michel ROUX, Sébastien TREMBLAY et Martin-Pierre LAVIGNE du Centre Eau, Terre et Environnement de l'INRS pour leur assistance technique à l'étude. Nous tenons également à remercier les réviseurs anonymes pour leurs commentaires constructifs. Ceux-ci nous ont permis d'améliorer grandement cette communication. Ce travail de recherche et de développement s'inscrit dans le cadre du projet GIBSI II, subventionné par le Programme d'aide à la recherche et au développement en environnement (PARDE) du ministère de l'Environnement du Québec.

\section{RÉFÉRENCES BIBLIOGRAPHIQUES}

ARNOLD J.G., WILLIAMS J.R., 1995. SWRRB - A Watershed Scale Model for Soil and Water Resources Management. Computer Models of Watershed Hydrology. Highlands Ranch, CO, V.P. Singh, Water Resources Publications, 847-908.

BROWN L.C., BARNWELL JR T.O., 1987. The Enhanced Stream Water Quality Models QUAL2 and QUAL2E-UNCAS: Documentation and User Manual. Athens, Georgia, US, Environmental Protection Agency, $189 p$.

CHEN C.W., GOLSTEIN R.A., HERR J., ZIEMELIS L., OLMSTED L., 1998. Uncertainty analysis for watershed management. Dans Proceedings of Watershed Management: Moving from Theory to Implementation, Specialty Conference Series. Water Environment Federation, Denver, CO, 3 au 6 mai, 1998, 1097-1104.

CPVQ, 1996. Grilles de référence en fertilisation. Conseil des Productions Végétales du Québec. Ministère de l'Agriculture et de l'Alimentation du Québec. Agdex 540. $128 \mathrm{p}$.

CURREY G.W., KLEINMAN J.I., 1999. Watershed-based permitting: a national pers- pective. Dans Environmental Permitting Symposium, Proceedings sponsored by the Air \& Waste Management Association, the Water Environment Federation and the American Academy of Environmental Engineers in Association with US EPA's Office of Air Quality Planning and Standards, Office of Wastewater Management, and Office of Solid Waste, Research Triangle Park, NC, 17-19 février, 1999, VIP-90, Vol. I, 361-371.

CRÉAC, 1995. Productions animales. AGDEX 400 , Comité de références économiques en agriculture du Québec.

FORTIN J.-P., MOUSSA R., BOCQUILLON C., VILLENEUVE J.-P., 1995. HYDRO$T E L$, un modèle hydrologique distribué pouvant bénéficier des données fournies par la télédétection et les systèmes d'information géographique. Revue des sciences de l'eau, 8, 97-124.

FORTIN J.-P., TURCOTTE R., MASSICOTTE S., MOUSSA R., FITZBACK J., VILLENEUVE J.-P., 2001a. A distributed watershed model compatible with remote sensing and GIS data. Part 1: description of the model. Journal of Hydrologic Engineering, 6 (2), 91-99. 
FORTIN J.-P., TURCOTTE R., MASSICOTTE S., MOUSSA R., FITZBACK J., VILLENEUVE J.-P., 2001b. A distributed watershed model compatible with remote sensing and GIS data. Part 2: application to Chaudière watershed. Journal of Hydrologic Engineering, 6 (2), 100-108.

GANGBAZO G., 1996. Expériences de contrôle de la pollution diffuse agricole à l'échelle du bassin versant. Vecteur Environnement, 29 (2), 65-71.

GANGBAZO G., 1997. Contrôle de la pollution diffuse agricole par l'approche des objectifs environnementaux de rejet. Vecteur Environnement, 30 (4), 25-31.

GANGBAZO G., PAINCHAUD J., 1999. Incidence des politiques et programmes d'assainissement agricole sur la qualité de l'eau de six rivières, 1988-1995. Vecteur Environnement, 32 (1), 29-36.

GANGBAZO G., CLUIS D., BERNARD C., 1999. Connaissance acquise en pollution diffuse agricole au Québec, 1993-1998: analyse et perspective. Vecteur Environnement, 32 (4), 36-45.

GARIÉPY S., ROUSSEAU A.N, 2000. La gestion de l'eau par bassin versant aux ÉtatsUnis. Vecteur Environnement, 33 (5), 43-50.

GOUIN D., 1984. La détermination des objectifs de traitement dans un projet d'assainissement. Sciences et Techniques de l'Eau, 17 (4), 383-388.

Gouvernement du Québec, 1997. Symposium sur la gestion de l'eau au Québec. Document de référence. Bibliothèque nationale du Québec.

HAITH D.A., 1982. Environmental Systems Optimization. John Wiley \& Sons. New York, NY.

HESSION W.C., STORM D.E., 2000. Watershed-level uncertainties: implications for phosphorus management and eutrophication. Journal of Environmental Quality, 29, 1172-1179.

JARRELL W.M., 1999. Getting Started with TMDLs. YSI Incorporated, Environmental Products Group (www.ysi.com).

LAVIGNE M.-P., ROUSSEAU A.N., TURCOTTE R., VILLENEUVE J.-P., 2002. Détermination de l'impact du déboisement à grande échelle sur le régime hydrologique d'un bassin versant de
$728 \mathrm{~km}^{2}$ à l'aide d'un système de modélisation intégrée. (Article proposé au : Canadian Journal of Civil Engineering).

MAILHOT A., ROUSSEAU A.N., MASSICOTTE S., DUPONT J., VILLENEUVE J.P., 1997. A Watershed-Based System for the Integrated Management of Surface Water Quality: The GIBSI System. Water Science Technology, 36 (5), 381-387.

MICHAUD A., DESLANDES J., LAUZIER R., ENRIGHT P., 2001. L'indice de risque des pertes en phosphore : un outil diagnostique des systèmes de production agricole. Dans Comptes rendus Colloque en agroenvironnement: l'agriculture et l'environnement en harmonie, Centre de référence en agriculture et agroalimentaire du Québec (CRAAQ), Drummondville, 21-22 novembre 2001.

Ministère de l'Environnement du Québec, 1991 (rév, 2001). Méthode de calcul des objectifs environnementaux de rejet pour les contaminants du milieu aquatique (1991 ; rév. 1994 ; rév. 1996 ; rév. 2001). Ministère de l'Environnement du Québec, Québec.

Ministère de l'Environnement du Québec, 1990 (rév. 1992). Critères de qualité de l'eau (1990 ; rév. 1992). Service de l'évaluation des rejets toxiques et Direction de la qualité des cours d'eau, ministère de l'Environnement du Québec, Québec.

Ministère de l'Environnement et de la Faune du Québec, 1995. Comité de travail sur les objectifs de qualité de l'eau pour l'assainissement agricole. Direction des écosystèmes aquatiques et Direction des politiques des secteurs agricole et naturel, Ministère de l'Environnement et de la Faune du Québec, Québec.

NOLET J., NOLET P., ROY L., DROLET R., VILLENEUVE S., 1999. Enjeu sur l'état du Saint-Laurent - La contribution des activités agricoles à la détérioration du Saint-Laurent. Équipe conjointe bilan, composée de représentants d'Environnement Canada, de Pêches et Océans Canada et du ministère de l'Environnement du Québec. Environnement Canada, Centre Saint-Laurent.

NOVOTNY V., 1999. Integrating diffuse/nonpoint source of pollution and water body restoration into watershed management. Journal of the American Water Resources Association, 35 (4), 717-727. 
NOVOTNY V., OLEM H., 1994. Water Quality: Prevention, Identification and Management of Diffuse Pollution. VanNostrandReinhold, New York, NY.

Official Journal of the European Communities, 2000. Directive 2000/60/EC of the European Parliament and of the Council of 23 October establishing a framework for Community action in the field of water policy. OJ C L 327, 22 December 2000.

PAINCHAUD J., 1997a. La qualité de l'eau des rivières du Québec - État et tendances. Ministère de l'Environnement et de la Faune, Direction des écosystèmes aquatiques, Québec.

PAINCHAUD J., 1997b. Tendances de la qualité de l'eau des rivières du Québec, 1979-1994. Vecteur Environnement, 30 (2), 43-50.

RENARD K.G., FOSTER G.R., WEESIES G.A., McCOOL D.K., YODER D.C., 1997. Predicting Soil Erosion by Water: $A$ Guide to Conservation Planning with the Revised Universal Soil Loss Equation (RUSLE). Agricultural Handbook 703, US Department of Agriculture, Agricultural Research Service, Washington DC, 404.

ROUSSEAU A.N, MAILHOT A., VILLENEUVE J.-P., 2000a. La gestion intégrée de l'eau par bassin versant : Problématique, éléments de solution et exemples d'application du système informatisé GIBSI. Vecteur Environnement, 33 (5), 27-30 et 51-54.

ROUSSEAU A.N., MAILHOT A., TURCOTTE R., DUCHEMIN M., BLANCHETTE C., ROUX M., DUPONT J., VILLENEUVE J.P., 2000b. GIBSI: an integrated modelling system prototype for river basin management. Hydrobiologia, 422-423, 465-475.

SHARPLEY A.N., WILLIAMS J.R., 1990 EPIC - Erosion/Productivity Impact Calculator: 1 Model Documentation. US Department of Agriculture Technical Bulletin $n^{\circ} 1768,235$.

SHOEMAKER L., LAHLOU M., BRYER M., KUMAR D., KRATT K., 1997. Compendium of Tools for Watershed Assessment and TMDL Development. EPA 841-B-97006, United States Environmental Protection Agency, Office of Water (4305), Washington, DC.
SIMONEAU M., PELLETIER L., MARTEL N., 1998. Le bassin versant de la rivière Chaudière : profil géographique, sources de pollution et interventions d'assainissement, pages 1.1 à 1.34 , dans ministère de l'Environnement et de la Faune (ed.), Le bassin versant de la rivière Chaudière : l'état de l'écosystème aquatique, 1996. Direction des écosystèmes aquatiques, Québec, envirodoq no EN980022.

Statistiques Canada, 1999. Le Canada en statistiques (CANSIM, matrices 6367 à 6379). Ottawa : Statistiques Canada. [Disponible en ligne à : http ://www.statcan.ca/francais/Pgdb/People/Population/demo02_f.htm]

TURCOTTE R., Fortin J.-P., Rousseau A.N., Massicotte S., Villeneuve J.-P., 2001. Determination of the drainage structure of a watershed using a digital elevation model and a digital river and lake network. Journal of Hydrology, 240, 225242.

US EPA, 1997. Technical Guidance Manual for Developing Total Maximum Daily Loads, Book II: Streams and Rivers, Part I: Biochemical Oxygen Demand/Dissolved Oxygen and Nutrients/Eutrophication. EPA 823-B-97-002, United States Environmental Protection Agency, Office of Water (4305), Washington, DC 20460.

US EPA, 1999. Protocol for Developing Nutrient TMDLs. EPA . 841-B-99-007. Office of Water (4503F), United States Environmental Protection Agency, Washington DC, 135.

VILLENEUVE J.-P., HUBERT P., MAILHOT A., ROUSSEAU A.N., 1998a. La modélisation hydrologique et la gestion de l'eau. Revue des sciences de l'eau, 11 (no spécial), 19-40.

VILLENEUVE J.-P., BLANCHETTE C., DUCHEMIN M., GAGNON J.-F., MAILHOT A., ROUSSEAU A.N., ROUX M. TREMBLAY J.-F., TURCOTTE R., 1998b. Rapport Final du Projet GIBSI, Mars 1998, Rapport n R-462, INRS-Eau, Sainte-Foy, QC.

VILLENEUVE J.-P., BLANCHETTE C., DUCHEMIN M., MAILHOT A., ROUSSEAU A.N., ROUX M., TRÉPANIER S., TURCOTTE R., 1998c. Document relatif aux commentaires du Comité de suivi du Projet GIBSI, Décembre 1998, Rapport $\mathrm{n}^{\circ} \mathrm{R}-462$ Add1, INRS-Eau, Sainte-Foy, $\mathrm{QC}$. 
WISCHMEIER W.H., SMITH D.D., 1978. Predicting Rainfall Erosion Losses - A Guide to Conservation Planning. Agricultural Handbook $n^{\circ} 537$, US Department of Agriculture, Washington, DC, 58.
YULIANTI J.S., LENCE B.J., JOHNSON G.V., TAKYI A.K., 1999. Non-point source water quality management under input information uncertainty. Journal of Environmental Management, 55, 199-217. 